\title{
Surface reaction-diffusion kinetics on lattice at the microscopic scale
}

\author{
Wei-Xiang Chew, ${ }^{1,2}$ Kazunari Kaizu, ${ }^{1}$ Masaki Watabe, ${ }^{1}$ Sithi V. Muniandy, ${ }^{2}$ Koichi Takahashi, ${ }^{1}$ and Satya N. V. Arjunan ${ }^{1, *}$ \\ ${ }^{1}$ Laboratory for Biologically Inspired Computing, RIKEN Center for Biosystems Dynamics Research, Suita, Osaka, Japan \\ ${ }^{2}$ Department of Physics, Faculty of Science, University of Malaya, 50603, Kuala Lumpur, Malaysia
}

(Received 17 November 2018; revised manuscript received 30 January 2019; published 19 April 2019)

\begin{abstract}
Microscopic models of reaction-diffusion processes on the cell membrane can link local spatiotemporal effects to macroscopic self-organized patterns often observed on the membrane. Simulation schemes based on the microscopic lattice method (MLM) can model these processes at the microscopic scale by tracking individual molecules, represented as hard spheres, on fine lattice voxels. Although MLM is simple to implement and is generally less computationally demanding than off-lattice approaches, its accuracy and consistency in modeling surface reactions have not been fully verified. Using the Spatiocyte scheme, we study the accuracy of MLM in diffusion-influenced surface reactions. We derive the lattice-based bimolecular association rates for two-dimensional (2D) surface-surface reaction and one-dimensional (1D) volume-surface adsorption according to the Smoluchowski-Collins-Kimball model and random walk theory. We match the time-dependent rates on lattice with off-lattice counterparts to obtain the correct expressions for MLM parameters in terms of physical constants. The expressions indicate that the voxel size needs to be at least $0.6 \%$ larger than the molecule to accurately simulate surface reactions on triangular lattice. On square lattice, the minimum voxel size should be even larger, at $5 \%$. We also demonstrate the ability of MLM-based schemes such as Spatiocyte to simulate a reaction-diffusion model that involves all dimensions: three-dimensional (3D) diffusion in the cytoplasm, 2D diffusion on the cell membrane, and 1D cytoplasm-membrane adsorption. With the model, we examine the contribution of the $2 \mathrm{D}$ reaction pathway to the overall reaction rate at different reactant diffusivity, reactivity, and concentrations.
\end{abstract}

DOI: 10.1103/PhysRevE.99.042411

\section{INTRODUCTION}

Many essential and intriguing intracellular biochemical systems are mediated by the cell membrane. These systems include cell polarity establishment [1], symmetrical cell division [2], modulation of signal transduction [3], and directed cell migration [4]. Spatiotemporal patterns arising from protein self-organization on the membrane [5], play a central role in these systems. The proteins self-organize primarily by reaction and diffusion processes. Membrane interactions can be classified as surface-surface reactions, where membranebound molecules react with each other, and volume-surface reactions, where cytosolic molecules react with membrane lipids or membrane-bound molecules.

To uncover the mechanisms underlying these systems, reaction-diffusion modeling approaches have been useful [6-10]. In general, the choice of modeling approach depends on the time and length scales of the system $[11,12]$. When the molecule copies are abundant and are well mixed in the surface compartment, macroscopic modeling approaches that apply rate [13] or reaction-diffusion [13,14] equation,

\footnotetext{
*satya@ riken.jp
}

Published by the American Physical Society under the terms of the Creative Commons Attribution 4.0 International license. Further distribution of this work must maintain attribution to the author(s) and the published article's title, journal citation, and DOI. are sufficient. If the molecule number is small or when the molecules are not homogeneously distributed in the compartment, mesoscopic methods based on the reaction-diffusion master equation (RDME) [15-18] can be employed since they account for both the fluctuations from a small number of molecules and the spatial inhomogeneity across well mixed compartment subvolumes [19,20].

Although macroscopic and mesoscopic approaches are applicable for large scale simulations, the well mixed assumption imposes several limitations. These approaches, for example, cannot explicitly capture the effects of space at the microscopic scale that arise from the interactions of finitesized molecules [21-23], fast rebinding of reactants [24-26], and microscopic surface inhomogeneity such as lipid domains and membrane-associated cytoskeletal structures [21,27-30]. The spatial effects can alter not only the diffusion behavior [31-33], but also the reaction kinetics [22,28-30,34], leading to different physiological outcomes. For example, clustering of membrane receptors changes the response of signaling network [26], fluctuation in protein copy number promotes cell polarization in the absence of spatial cue [6], and rapid protein rebinding affects spatiotemporal patterns on the membrane [25] and amplifies noise during ligand interactions [35]. Moreover, macroscopic and mesoscopic approaches adopt the macroscopic reaction rate constant for all reactions, which is not sufficient for irreversible bimolecular surfacesurface and one-dimensional volume-surface reactions because these time-dependent reaction rates do not reach steady state [36,37]. 
Microscopic approaches are more suitable to model surface interactions for short time scales when the microscopic spatial effects need to be directly accounted for [38-42]. In off-lattice microscopic particle-based methods, diffusion is simulated in continuous space with Gaussian distributed displacement. Bimolecular reactions are executed according to the Smoluchowski-Collins-Kimball (SCK) $[43,44]$ or the Doi [45-47] physical model. In the former, the reaction occurs either immediately or with a probability of reflection when the distance between reactants equals to a predefined reaction radius, whereas in the Doi model, the reaction occurs with a fixed probability per unit time when the reactants are closer than the radius. Off-lattice SCK methods that support surface reactions include Smoldyn [48,49], CDS [50], and eGFRD [51], while the Doi model is adopted by MCell [52], ReaDDy [53,54], and SpringSaLaD [55]. Smoldyn also recently included the option to support the Doi model. All of these methods except MCell can simulate volume occupying molecules. In a recent performance benchmark that did not include CDS [42], Smoldyn displayed the fastest simulation run time for a simple enzymatic reaction model in volume compartment.

Schemes based on the microscopic lattice method ${ }^{1}$ (MLM) $[39,56]$ attempt to reduce the cost of resolving molecular collisions by discretizing the space into fine molecule-sized voxels. In the Spatiocyte scheme, for example, a molecule only checks its destination voxel for occupancy before performing a bimolecular reaction with the occupying molecule or moving into it if it is vacant [56,57]. Consequently, Spatiocyte exhibits better run time and scaling performances than Smoldyn when diffusing volume occupying molecules [56]. The run time of Spatiocyte is also comparable to Smoldyn in the benchmark enzymatic reaction model [56]. The reduced computational cost and the simplicity of MLM implementation have promoted its applications in both biological [38,57-61] and chemical [62-64] surface reactions. Nonetheless, biological surfaces such as cell and nuclear membranes are not arranged as fixed lattice structures. Further, since diffusion and reaction kinetics can be influenced by the lattice arrangement [65-67], the accuracy of MLM compared to off-lattice particle-based methods requires careful examination. Notably, a consistent approach is needed to determine MLM parameters such as voxel size and on-lattice reaction probability that can replicate the kinetics in continuous space.

In previous work, the SCK model was used together with the Spatiocyte scheme to construct a general theoretical framework of MLM for simulating reaction and diffusion processes in three-dimensional (3D) space [56]. Within this framework, the expressions for on-lattice reaction rate constant, reaction and rebinding probabilities, and voxel size were derived to reproduce off-lattice reaction kinetics consistently. Here, we extend this framework for two-dimensional (2D) surface-surface and one-dimensional (1D) volume-surface reactions. We also employ the SCK model to derive on-lattice time-dependent rate coefficients for the surface reactions. We then obtain the expressions for the MLM parameters by equating the off-lattice rate equations with the on-lattice

\footnotetext{
${ }^{1}$ Also called Monte Carlo lattice gas model [39].
}

counterparts. In Sec. II of this paper, we introduce the existing continuum-based reaction kinetics theory for surface reactions. In Sec. III, we derive the expressions for surface reaction rates on lattice according to the Spatiocyte scheme and verify them using the continuum theory. In Sec. IV, we demonstrate the applicability of the derived expressions for surface reactions that involve all dimensions. We also look at the contribution of $3 \mathrm{D}$ and $2 \mathrm{D}$ reaction pathways to the overall reaction rate. Finally in Sec. V, we discuss the implications and limitations of this work.

\section{CONTINUUM-BASED REACTION KINETICS THEORY}

Consider a many-body bimolecular reaction,

$$
A+B \rightarrow B
$$

with $A$ and $B$ having radii $r_{A}$ and $r_{B}$, and diffusion coefficients $D_{A}$ and $D_{B}$, respectively. According to the SCK model, when the distance between a pair of $A$ and $B$ molecules is the sum of their radii $R=r_{A}+r_{B}$, the two will react with an intrinsic rate $k_{a}$. The fraction of $A$ remaining in the system is described by the survival probability, $S_{\mathrm{irr}, \mathrm{A}}(t)=[A(t)] /[A(0)]$, where [ ] denotes the concentration. When $[B(0)] \gg[A(0)]$, the survival probability of $A$ is provided in the rate equation [36]:

$$
\frac{d S_{\mathrm{irr}, \mathrm{A}}(t)}{d t}=-[B] k(t) S_{\mathrm{irr}, \mathrm{A}}(t),
$$

where $k(t)$ represents the time-dependent rate coefficient. The solution for the survival probability requires the integration of the rate coefficient [Eq. (2.35) in Ref. [36]]:

$$
S_{\mathrm{irr}, \mathrm{A}}(t,[B])=\exp \left[-[B] \int_{0}^{t} k(\tau) d \tau\right]
$$

According to the particle-pair formalism of the SCK model [68-71], the many-body reaction can be approximated by a simpler two-body problem. The time-dependent rate coefficient can thus be expressed as the product of $k_{a}$ and the survival probability of an in-contact reactant pair:

$$
k(t)=k_{a}\left[1-\int_{0}^{t} p_{\mathrm{reb}}(R, \tau \mid R, 0) d \tau\right] .
$$

Here, $p_{\text {reb }}(R, \tau \mid R, 0)$ specifies the rebinding-time probability distribution for a reactive particle-pair separated by a distance $R$ at time $\tau$, given that the pair was initially in contact.

The specific functional form of the rate coefficient depends on the spatial dimension of the reactant diffusion. The dimension is $2 \mathrm{D}$ for surface-surface reactions, whereas for volume-surface reactions, it is determined by the target reactant of the cytosolic molecule. The dimension is 3D when the reactant is a membrane-associated molecule or $1 \mathrm{D}$ when the cytosolic molecule reacts directly with the lipid membrane. For clarity, we refer to the $1 \mathrm{D}$ volume-surface reaction as adsorption. Since we have previously described the theory for $3 \mathrm{D}$ reactions [56], in the following subsections we provide the theory for $2 \mathrm{D}$ surface-surface reaction and $1 \mathrm{D}$ volume-surface adsorption. 


\section{A. 2D surface-surface reaction}

\section{Irreversible reaction}

The time-dependent rate coefficient for 2D association reaction with radiation boundary condition is given in the Laplace form as [36]

$$
k_{2 D}(s)=\frac{k_{a 2 D}}{s\left[1+k_{a 2 D} \widetilde{g}(s)\right]} .
$$

Here, $k_{a 2 D}$ is the intrinsic rate constant with dimensions of length $\mathrm{L}$ and time $\mathrm{T}$, given by $\mathrm{L}^{2} \mathrm{~T}^{-1}$, and $\widetilde{g}(s)$ is the Green's function for 2D diffusion defined as [72]

$$
\widetilde{g}(s)=\frac{K_{0}(\tilde{s})}{2 \pi D \tilde{s} K_{1}(\tilde{s})} .
$$

$K_{0}$ and $K_{1}$ are the modified Bessel functions of the second kind, $\tilde{s}=\sqrt{s R^{2} / D}$, and $D=D_{A}+D_{B}$.

Equation (5) can thus be written as

$$
k_{2 D}(s)=2 \pi D \tilde{s} \frac{K_{1}(\tilde{s})}{s\left[K_{0}(\tilde{s})+2 \pi \tilde{s} K_{1}(\tilde{s}) / \kappa\right]},
$$

with $\kappa=k_{a} / D$. In the limit of small $\tilde{s}$, we can approximate the modified Bessel functions:

$$
\tilde{s} K_{1}(\tilde{s}) \approx 1-\frac{2 \ln \left(\tilde{s} e^{\gamma} / 2\right)+1}{4} \tilde{s}^{2}+O\left(\tilde{s}^{4}\right),
$$

and

$$
K_{0}(\tilde{s}) \approx-\ln \left(\tilde{s} e^{\gamma} / 2\right)-\frac{2 \ln \left(\tilde{s} e^{\gamma} / 2\right)+1}{4} \tilde{s}^{2}+O\left(\tilde{s}^{4}\right),
$$

where $\gamma=0.5772156$ is the Euler constant.

Using these approximations, the asymptotic expansion of Eq. (7) can be expressed as

$$
\begin{aligned}
\lim _{s \rightarrow 0} s k_{2 D}(s)= & \frac{4 \pi D}{-2 \ln [R \sqrt{s / D}]+\ln 4+\ln [\exp 2(4 \pi / \kappa-\gamma)]} \\
& +O(s) \\
= & \frac{4 \pi D}{\ln \left[C_{c} / s\right]}+O(s),
\end{aligned}
$$

with $C_{c}=4 D \exp (4 \pi / \kappa-2 \gamma) / R^{2}$. The corresponding longtime approximation is given as [73]

$$
\begin{aligned}
k_{2 D}\left(t \gg \frac{R^{2}}{D}\right)= & 4 \pi D\left(\frac{1}{\ln \left(C_{c} t\right)}-\frac{\gamma}{\left(\ln \left(C_{c} t\right)\right)^{2}}-\frac{1.311}{\left(\ln \left(C_{c} t\right)\right)^{3}}\right. \\
& \left.+\frac{0.25}{\left(\ln \left(C_{c} t\right)\right)^{4}}+O\left(t^{-1} \ln (t)^{-2}\right)\right)
\end{aligned}
$$

where the relative error to the exact form is less than $1 \%$ at $t=100 R^{2} / D$.

The slow logarithmic decay of $k_{2 D}(t)$ originates from the modified Bessel function and is fundamentally connected to the recurrence property of the random walk in $2 \mathrm{D}$. A random walker in 3D has an asymptotically finite chance to escape from the origin. However, in the lower dimensions (1D and 2D), the return probability is unity, as described by the Polya's theorem. In the case of $2 \mathrm{D}$, the first passage time of returning to the origin exhibits slow logarithmic decay [74], which in turn causes the slow decay in $k_{2 D}(t)$. As a result, unlike in the $3 \mathrm{D}$ reaction, the long-time rate coefficient in $2 \mathrm{D}$ does not converge to a constant term. This behavior has been observed in several experimental studies [22]. The lack of an apparent steady state and the slow decay in the $2 \mathrm{D}$ reaction rate have prompted the use of a time-dependent rate for the purposes of modeling and analysis of experiments $[22,75]$.

\section{Steady-state rate constant}

Szabo [36] provided an alternative way to define a steadystate rate constant by coupling the irreversible bimolecular reaction with the production of $A$ species. Following this strategy, a steady-state expression can be defined in terms of the mean lifetime of $A, \tau_{m}$ [36]:

$$
\begin{aligned}
k_{s s} & =\frac{1}{[B] \tau_{m}}=\left[[B] \int_{0}^{\infty} S_{\mathrm{irr}, \mathrm{A}}(t) d t\right]^{-1} \\
& =\left[[B] \int_{0}^{\infty} \exp \left(-[B] \int_{0}^{t} k_{2 D}(\tau) d \tau\right) d t\right]^{-1} .
\end{aligned}
$$

$k_{s s}$ can be evaluated using the mean-field self-consistency condition [36]:

$$
k_{s s}=[s \hat{k}(s)]_{s=k_{s s}[B]} .
$$

Substituting the asymptotic form of $k_{2 D}(s)$, as defined in Eq. (10), into Eq. (13) yields

$$
\frac{k_{s s}}{2 \pi D} \approx\left[\ln 2-\gamma-\ln \left[R \sqrt{k_{s s}[B] / D}\right]+1 / \kappa\right]^{-1} .
$$

Rewriting some variables in terms of the molecule area fraction $\phi=\pi R^{2}[B]$, and taking the small concentration limit $\phi \rightarrow 0$ gives the following approximation:

$$
\begin{aligned}
\frac{k_{s s}}{2 \pi D} & \approx[\ln 2-\gamma-\ln [\sqrt{2 \phi y}]+1 / \kappa]^{-1} \\
& \approx\left[\frac{1}{2} \ln 2-\gamma-\frac{1}{2} \ln \phi+1 / \kappa\right]^{-1} .
\end{aligned}
$$

Finally, the steady-state rate constant for the radiation boundary condition is obtained as

$$
k_{s s 2 D} \approx \frac{4 \pi D}{\ln 2-2 \gamma-\ln \phi+4 \pi / \kappa} .
$$

Similar to the $3 \mathrm{D}$ effective rate constant in $1 / k_{s s 3 D}=1 / k_{a}+$ $1 /(4 \pi R D)$, the $2 \mathrm{D}$ steady-state rate constant depends on the intrinsic rate $k_{a}$ and the relative diffusion coefficient $D$. Interestingly, the $2 \mathrm{D}$ rate constant has the additional dependency on the concentration of $B$. The steady-state constant given in Eq. (16) is an extension of the solution based on the absorbing boundary condition provided in Eq. (4.10) of Ref. [36].

\section{Reversible reaction}

In the SCK model for 2D reversible reaction,

$$
A+B \underset{k_{d 2 D}}{\stackrel{k_{a 2 D}}{\rightleftharpoons}} A B,
$$

a bound pair $A-B$ dissociates with the rate constant $k_{d 2 D}$ $\left(\mathrm{T}^{-1}\right)$ into $A$ and $B$, separated at distance $R$. The survival probability of $A$, defined as $S_{\mathrm{rev}, A}(t)$, can be calculated using the first variant of the multiparticle kernel theory (MPK1) $[76,77]$. Although the closed form solution for $S_{\text {rev }, A}(t)$ in $2 \mathrm{D}$ is not available, it can be evaluated by numerically solving the 
normalized deviation defined as

$$
\frac{S_{\mathrm{rev}, A}(t)-S_{\mathrm{rev}, A}(\infty)}{S_{\mathrm{rev}, A}(0)-S_{\mathrm{rev}, A}(\infty)}=\mathcal{L}^{-1}\left[\frac{1}{s+\lambda \widetilde{F}(s)}\right] .
$$

Here, the term,

$$
\widetilde{F}(s)=\frac{k_{d 2 D}}{\lambda} \widetilde{F}_{\mathrm{gem}}(s)+\frac{[B] k_{a 2 D}}{\lambda} \widetilde{F}_{\mathrm{irr}}\left(s ;[B]^{\prime}\right),
$$

is the diffusion factor function, $S_{\mathrm{rev}, A}(\infty)=k_{d 2 D} / \lambda=1 /(1+$ $\left.[B] k_{a 2 D} / k_{d 2 D}\right)$ is the equilibrium value, $\lambda=k_{d 2 D}+[B] k_{a 2 D}$ is the chemical kinetics relaxation rate constant, and $[B]^{\prime}=$ $\lambda / k_{a 2 D}$ is the modified concentration. $\widetilde{F}_{\text {gem }}(s)=1+k_{a 2 D} \widetilde{g}(s)$ contains the 2D Green's function term $\widetilde{g}(s)$ as given in Eq. (6), whereas the function,

$$
\widetilde{F}_{\text {irr }}\left(s ; c_{0}\right)=\frac{c_{0} k_{a 2 D} \widetilde{S}_{\text {irr,A }}\left(s ; c_{0}\right)}{1-s \widetilde{S}_{\mathrm{irr}, \mathrm{A}}\left(s ; c_{0}\right)},
$$

uses the term $\widetilde{S}_{\text {irr }, A}\left(s ; c_{0}\right)$, which is the Laplace transform of the irreversible reaction survival probability $S_{\mathrm{irr}, A}\left(t ; c_{0}\right)$.

\section{B. 1D volume-surface adsorption}

Before describing the rate for volume-surface adsorption, we first consider the 1D SCK model, where a single immobile species $B$ interacts with multiple mobile species $A$ on a filament according to Eq. (1). Species $A$ can collide with species $B$ from both sides of $B$, while there is no self-interaction among $A$ molecules. The time-dependent rate coefficient of this reaction with radiation boundary condition is given as [36]

$$
k_{1 D}(t)=k_{s a} \exp \left(\kappa^{2} D t / 4\right) \operatorname{erfc}(\kappa \sqrt{D t / 4}),
$$

with $\kappa=k_{a} / D$ denoting the ratio between the intrinsic adsorption rate constant $k_{a}=k_{s a}$ (unit $\mathrm{LT}^{-1}$ ) and the relative diffusion coefficient $D$. At long time, Eq. (21) behaves asymptotically as

$$
k_{1 D}(t \rightarrow \infty) \approx 2 \sqrt{\frac{D}{\pi t}}+O\left(t^{-3 / 2}\right)
$$

Next we consider a volume-surface adsorption system that involves an adsorbing plane at $x=0$ and bulk molecules at $x>0$. Initially, the molecules of concentration $c_{0}$ are distributed uniformly in the bulk and are absent on the surface. For a surface adsorption process that follows the radiation boundary condition, the number of molecules adsorbed to the surface varies as [Eq. (3.37) of Ref. [78]]

$$
N_{s}(t)=\frac{c_{0} S}{\kappa}\left\{\exp \left(\kappa^{2} D t\right) \operatorname{erfc}(\kappa \sqrt{D t})-1+2 \kappa \sqrt{D t / \pi}\right\},
$$

where $S$ is the area of the plane. The corresponding adsorption rate is well described by the time-dependent adsorption rate coefficient $k_{s a}\left(t^{\prime}\right)$ :

$$
\frac{d N_{s}\left(t^{\prime}\right)}{d t}=k_{s a}\left(t^{\prime}\right) c_{0} S .
$$

Note that the adsorption rate coefficient differs from the 1D SCK rate by a factor of two: $k_{s a}\left(t^{\prime}\right)=k_{1 D}(t) / 2$, because in the latter it occurs on both sides of the plane. At long time, the adsorption rate coefficient becomes

$$
k_{s a}(t \rightarrow \infty) \approx \sqrt{\frac{D}{\pi t}}+O\left(t^{-3 / 2}\right) .
$$

As the bulk molecules are adsorbed to the surface, a spatial concentration gradient develops in the bulk. The spatialtemporal concentration profile of the bulk molecules $C(x, t)$ follows Eq. (3.35) of Ref. [78]:

$$
C(x, t)=c_{0}\left[\operatorname{erfc} \frac{x}{2 \sqrt{D t}}-\exp \left(\frac{k_{s a} x}{D}+\frac{k_{s a}^{2} t}{D}\right) \operatorname{erfc}\left(\frac{x}{2 \sqrt{D t}}+k_{s a} \sqrt{\frac{t}{D}}\right)\right] .
$$

When adsorbed molecules can dissociate from the surface with a rate $k_{s d}\left(\mathrm{~T}^{-1}\right)$, their number varies according to [Eq. (A.12) in Ref. [48]]

$$
N_{s}(t)=\frac{c_{0} S k_{s a} t\left[c_{1} \exp \left(c_{2}^{2}\right) \operatorname{erfc}\left(c_{2}\right)-c_{1}+c_{2} \exp \left(c_{1}^{2}\right) \operatorname{erfc}\left(c_{1}\right)-c_{2}\right]}{c_{1} c_{2}\left(c_{2}-c_{1}\right)},
$$

where

$$
c_{1}=\frac{k_{s a}-\sqrt{k_{s a}^{2}-4 D k_{s d}}}{2 \sqrt{D}} \sqrt{t},
$$

and

$$
c_{2}=\frac{k_{s a}+\sqrt{k_{s a}^{2}-4 D k_{s d}}}{2 \sqrt{D}} \sqrt{t} .
$$

\section{MICROSCOPIC LATTICE METHOD}

In this section, we derive the on-lattice rate coefficients for 2D surface-surface reaction and 1D volume-surface adsorption based on the Spatiocyte scheme [56,57]. A detailed description of the scheme in 3D is given in Sec. IIB and Algorithm I in Ref. [56]. For surface reactions, the scheme remains the same as in the $3 \mathrm{D}$ case except that molecules diffuse on the triangular lattice. In brief, a molecule of species $x$ hops randomly to one of its six nearest neighbor voxels at each diffusion time step $t_{d}=l^{2} / 4 D_{x}$, where $l$ is the voxel size (diameter) and $D_{x}$ is the species diffusion coefficient. When the destination voxel is occupied by a molecule belonging to a nonreactive species, the move will be rejected because of volume exclusion. If the destination voxel contains a reactant molecule, the corresponding reaction will occur with probability $P_{a}$; otherwise, if the reaction fails, the molecule will 
remain in its original voxel. When a molecule dissociates into two product molecules, the pair will be placed side-by-side on the lattice. In volume-surface adsorption, a 3D species will absorb to the surface with some probability upon collision with the surface voxel. The simulation scheme for surface reactions has been implemented in the Spatiocyte module of the E-CELL SYSTEM, Version 4, software [79].

The particle-pair formalism of the SCK model and random walk theory will be used in the derivation of on-lattice rate. In MLM, the SCK rate coefficient in Eq. (4) is discretized into [see Sec. II.C of Ref. [56] for derivation]

$$
k_{m}=k_{a}^{\prime}\left[1-\sum_{n=0}^{m} H_{n}\left(s_{0} \mid s_{1}\right)\right] \text {, for } m, n \in \mathbb{N} .
$$

$k_{a}^{\prime}$ is the initial lattice rate constant (see Appendix A), $H_{n}\left(s_{0} \mid s_{1}\right)$ is the rebinding-time probability distribution in diffusion step $n$, and $m$ is the simulation step. Rebinding here refers to the next reaction event of an in-contact reactant pair on lattice, $s_{0}$ denotes the voxel at the origin, and $s_{1}$ refers to an element from the set of immediate neighbor voxels of $s_{0}$. The rebinding-time probability is a function of random walk quantities such as $P_{n}\left(s_{a} \mid s_{b}\right)$, the voxel occupation probability from voxel $s_{b}$ to voxel $s_{a}$, that is, the probability of being at voxel $s_{a}$ after $n$ steps, given that the walk started at voxel $s_{b}$; and $F_{n}\left(s_{a} \mid s_{b}\right)$, the first-passage time distribution from voxel $s_{b}$ to $s_{a}$, that is, the probability of arriving at $s_{b}$ for the first time on the $n$th step, given that the walk started at voxel $s_{a}$. These quantities depend on the lattice arrangement, dimension of diffusion, and also the simulation scheme. The simulation step $m$ is related to the simulation time $t^{\prime}$ through the relation $2 d D_{x} t^{\prime}=m l^{2}$, where $d$ is the dimension of diffusion. In the following subsections of $2 \mathrm{D}$ and $1 \mathrm{D}$ reactions, we will derive the on-lattice rate coefficient based on the Spatiocyte simulation scheme.

\section{A. 2D surface-surface reaction}

\section{1. $2 D$ diffusion}

We first verify the diffusion behavior of molecules on the $2 \mathrm{D}$ triangular lattice by analyzing their mean squared displacement (msd). We simulated the random walks of the molecules on the lattice and log their trajectories to obtain the msd. Figure 1 shows that the log-log plot of the msd scales as $\left\langle r^{2}\right\rangle=4 D t$. In addition, we considered the influence of volume exclusion on the tracer molecules by introducing immobile obstacles on the lattice. The obstacle density is quantified by $\phi$, the ratio of the number of obstacles to the total number of surface voxels. At $\phi=0.3$, the msd scales nonlinearly at short time before becoming linear again at long time (circle marker in Fig. 1). As the obstacle density increases to the percolation threshold of the lattice at $\phi=$ 0.5 , the msd exhibits nonlinear scaling, which is a sign of anomalous diffusion [80].

\section{Irreversible reaction}

The methods presented in this work are generalized for any regular lattice arrangement, but we focus on the triangular lattice since it is used to simulate surface-surface reactions in the Spatiocyte scheme. The derivation of the rate coefficients

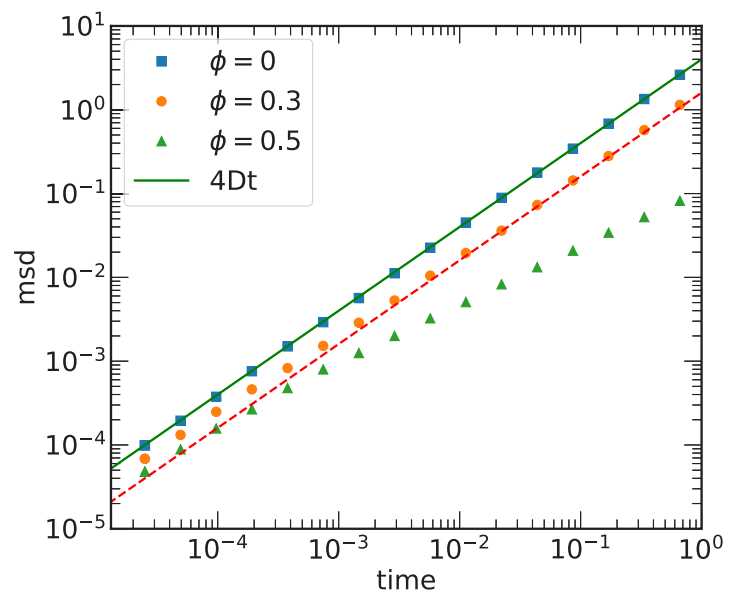

FIG. 1. Mean squared displacement (msd) of molecules on 2D lattice. Molecules perform random walk on triangular lattice with $\phi$ fraction of total surface voxels occupied by immobile obstacles. Green solid line represents the expected msd behavior for normal diffusion. Red dashed line denotes the linear scaling for $\phi=0.3$ at long time. Simulation was performed with voxel size $l=0.01 \mu \mathrm{m}$ in a square compartment of length $L=5 \mu \mathrm{m}$. Diffusion coefficient, $D=1 \mu \mathrm{m}^{2} \mathrm{~s}^{-1}$.

for activation-limited $\left(k_{a 2 D} \ll D\right)$ and diffusion-influenced $\left(k_{a 2 D} \gg D\right)$ reactions is treated separately because the simulation scheme executes these two types of reaction in a distinct manner.

In the activation-limited scheme, the generating function for the rebinding-time probability distribution $H_{n}\left(s_{0} \mid s_{1}\right)$ is given as (see Appendix B 1 in Ref. [56] for derivation)

$$
H\left(s_{0} \mid s_{1} ; z\right)=\frac{P_{a} F\left(s_{0} \mid s_{0} ; z\right)}{z+F\left(s_{0} \mid s_{0} ; z\right)\left(P_{a}-1\right)},
$$

where $P_{a}$ denotes the reaction probability. In terms of $P\left(s_{0} \mid s_{0} ; z\right)$, the generating function of $P_{n}\left(s_{0} \mid s_{0}\right), H\left(s_{0} \mid s_{1} ; z\right)$ becomes (see Appendix B 1 for full derivation)

$$
H\left(s_{0} \mid s_{1} ; z\right)=1-\frac{1}{P_{a} P\left(s_{0} \mid s_{0} ; z\right)\left[1+\frac{\left(1-P_{0}\right)}{P_{a} P\left(s_{0} \mid s_{0} ; z\right)}\right]} .
$$

The generating function $P\left(s_{0} \mid s_{0} ; z\right)$ for the triangular lattice in asymptotic form is given as (see Appendix B 2 for details)

$$
P\left(s_{0} \mid s_{0} ; z\right) \approx \frac{\sqrt{3}}{2 \pi} \ln \left[12(1-z)^{-1}\right]\{1+O(1-z)\} .
$$

By substituting Eq. (33) into Eq. (32), we obtain the following approximated form:

$$
H\left(s_{0} \mid s_{1} ; z\right) \approx-\frac{b_{1}}{P_{a}}\left\{\ln \left(\frac{E}{1-z}\right)\right\}^{-1},
$$

where $E=12 \exp \left\{b_{1}\left(1 / P_{a}-1\right)\right\}$ and $b_{1}=2 \pi / \sqrt{3}$. We then apply singularity analysis (Fig. VI.4 of Ref. [81]) on Eq. (34) to obtain the large $n$ behavior:

$$
H_{n}\left(s_{0} \mid s_{1}\right) \approx \frac{2 \pi}{\sqrt{3} P_{a}} \frac{1}{n}\left(\frac{1}{(\ln E n)^{2}}-\frac{2 \gamma}{(\ln E n)^{3}}+\frac{3 \gamma^{2}-\frac{\pi^{2}}{2}}{(\ln E n)^{4}}+\ldots\right) .
$$


With Eq. (35), we evaluate the discrete sum in Eq. (30) using the Euler-Mascheroni formula together with the definition of recurrence in $2 \mathrm{D}$ random walk: $\sum_{n=0}^{\infty} H_{n}=1$. The solution in high order $m$ terms is given by

$$
k_{m}^{2 D}=\frac{2 \pi k_{a 2 D}^{\prime}}{\sqrt{3} P_{a}}\left[\frac{1}{\ln E m}-\frac{\gamma}{(\ln E m)^{2}}+\frac{\gamma^{2}-\frac{\pi^{2}}{6}}{(\ln E m)^{3}}+\ldots\right] .
$$

Finally, we apply the definition of initial rate for the triangular lattice as given in Eq. (A5) and the relation $m l^{2}=4 D t^{\prime}$ to obtain the time-dependent rate coefficient:

$$
k_{2 D}^{\prime}(t)=4 \pi D\left[\frac{1}{\ln C_{l} t}-\frac{\gamma}{\left(\ln C_{l} t\right)^{2}}+\frac{\gamma^{2}-\frac{\pi^{2}}{6}}{\left(\ln C_{l} t\right)^{3}}+\ldots\right],
$$

where $C_{l}=48 D \exp \left\{b_{1}\left(1 / P_{a}-1\right)\right\} / l^{2}$.
In the derivation of the diffusion-influenced scheme, it is convenient to work with the Laplace form of Eq. (30):

$$
\hat{k}_{2 D}^{\prime}(s)=k_{a 2 D}^{\prime}[1-\hat{G}(s)] / s .
$$

Here $\hat{G}(s)$ is the Laplace form of the rebinding-time probability density on lattice, defined as [see Eq. (D79) in Ref. [56]]

$$
\hat{G}(s)=\beta_{1}\left[s+\beta-s F_{1}\left(s_{1} \mid s_{1}\right) z-\beta_{2} F\left(s_{1} \mid s_{1} ; z\right)\right]^{-1},
$$

where

$$
F\left(s_{1} \mid s_{1} ; z\right)=1-\frac{z^{2} P_{1}\left(s_{0} \mid s_{1}\right)}{P\left(s_{0} \mid s_{0} ; z\right)-1},
$$

$P_{1}\left(s_{0} \mid s_{1}\right)=1 / 6, \quad F_{1}\left(s_{1} \mid s_{1}\right)=1 / 3, \quad P_{1}\left(s_{2} \mid s_{1}\right)=1 / 2$, $z=\beta_{2} /\left(s+\beta_{2}\right), \beta=\beta_{1}+\beta_{2}, \beta_{1}=P_{a} / 6 t_{d}$, and $\beta_{2}=1 / t_{d}$. $t_{d}=l^{2} / 2 d D_{x}$ here refers to the average time interval needed for a molecule with diffusion coefficient $D_{x}$ to hop across one voxel. By applying the final value theorem, we get the asymptotic form for Eq. (38) as

$$
\begin{aligned}
\lim _{s \rightarrow 0} s \hat{k}_{2 D}^{\prime}(s) & =k_{a 2 D}^{\prime}\left[1-\lim _{s \rightarrow 0} \hat{G}(s)\right]=k_{a 2 D}^{\prime}\left[1-\frac{\beta_{1}}{\lim _{s \rightarrow 0}\left[s+\beta-s F_{1}\left(s_{1} \mid s_{1}\right) z-\beta_{2} F\left(s_{1} \mid s_{1} ; z\right)\right]}\right] \\
& =k_{a 2 D}^{\prime}\left[1-\left(1+\frac{\beta_{2}}{\beta_{1}} \lim _{z \rightarrow 1} \frac{z^{2} / 6}{P\left(s_{0} \mid s_{0} ; z\right)-1}\right)^{-1}\right] .
\end{aligned}
$$

Finally, by taking the small $z$ expansion together with Eq. (33), we obtain the asymptotic rate coefficient expression:

$$
\begin{aligned}
\lim _{s \rightarrow 0} s \hat{k}_{2 D}^{\prime}(s) & =\frac{2 \pi k_{a 2 D}^{\prime}}{P_{a} \sqrt{3}}\left\{\ln \left[\frac{12 \exp \left\{2 \pi\left(1 / P_{a}-1\right) / \sqrt{3}\right\}}{1-z}\right]\right\}^{-1} \\
& =\frac{4 \pi D}{\ln \left[E\left(1+\frac{4 D}{l^{2} s}\right)\right]} \\
& \approx \frac{4 \pi D}{\ln \left(C_{l} / s\right)} .
\end{aligned}
$$

By comparing the lattice and continuum rate coefficient, we found that the asymptotic expression in Eq. (42) for the diffusion-influenced scheme is the same as its continuum counterpart shown in Eq. (10), while the time domain expression in Eq. (37) for the activation-limited scheme is consistent with the continuum counterpart shown in Eq. (11). To match the lattice and continuum rates, we need to impose the equality $C_{l}=C_{c}$. It then implies that the reaction probability should be chosen as

$$
P_{a}=\left[1+\frac{\sqrt{3}}{2 \pi}\left(\ln \left(f^{2} / 12\right)+\frac{4 \pi D}{k_{a 2 D}}-2 \gamma\right)\right]^{-1},
$$

where $f=l / R$ denotes the ratio of voxel to the molecule size. Since probability $P_{a}$ is positive, it sets an additional constraint:

$$
\ln f+\frac{2 \pi}{\kappa}>-\frac{\pi}{\sqrt{3}}+\frac{\ln 12}{2}+\gamma=C_{1} .
$$

To satisfy the last inequality, $f=l / R$ has to be adapted according to the value of $\kappa$. Since $\kappa$ is always positive, we only need to set a lower bound expression for the voxel size:

$$
\begin{aligned}
& \ln f>C_{1}-\frac{2 \pi}{\kappa}>C_{1}, \quad f>\exp \left(C_{1}\right), \\
& l>\exp \left(C_{1}\right) R \approx 1.005887 R .
\end{aligned}
$$

In 3D MLM, accurate reaction kinetics requires the voxel size of hexagonal close-packed (hcp) lattice to be larger than the molecule by $l \approx 1.02086 R$ [56]. If an hcp lattice volume compartment is bounded by a triangular lattice surface, the $3 \mathrm{D}$ voxel size condition would still satisfy Eq. (45). Therefore, all surface and volume voxels in the model can adopt the same hcp voxel size.

The accuracy of the lattice theory can be verified by comparing the theoretical values for the rate coefficient $k_{2 D}^{\prime}(t)$ with the simulated values. We obtained the theoretical rate coefficient from the numerical inverse Laplace transform of Eq. (41). We simulated the reaction in Eq. (1) with Spatiocyte at both the activation-limited $(\kappa=0.01 \times 4 \pi)$ and the diffusion-limited $(\kappa=100 \times 4 \pi)$ regimes. We logged the number of surviving $A$ and used it to calculate the rate coefficient. The discretization of the time derivative in Eq. (2) gives the formula for discrete rate coefficient:

$$
k_{j+1}=-\frac{S_{j+2}-S_{j}}{[B] S_{j+1}\left(t_{j+2}-t_{j}\right)}, \quad \text { for } j \in \mathbb{Z}^{+},
$$

where $j$ is the index of the discretized $S_{A}$ and $t$. The boundary cases are computed as

$$
k_{1}=-\frac{S_{2}-S_{1}}{[B] S_{1}\left(t_{2}-t_{1}\right)}, \quad k_{N}=-\frac{S_{N}-S_{N-1}}{[B] S_{N}\left(t_{N}-t_{N-1}\right)},
$$

where $N$ denotes the final time step. 

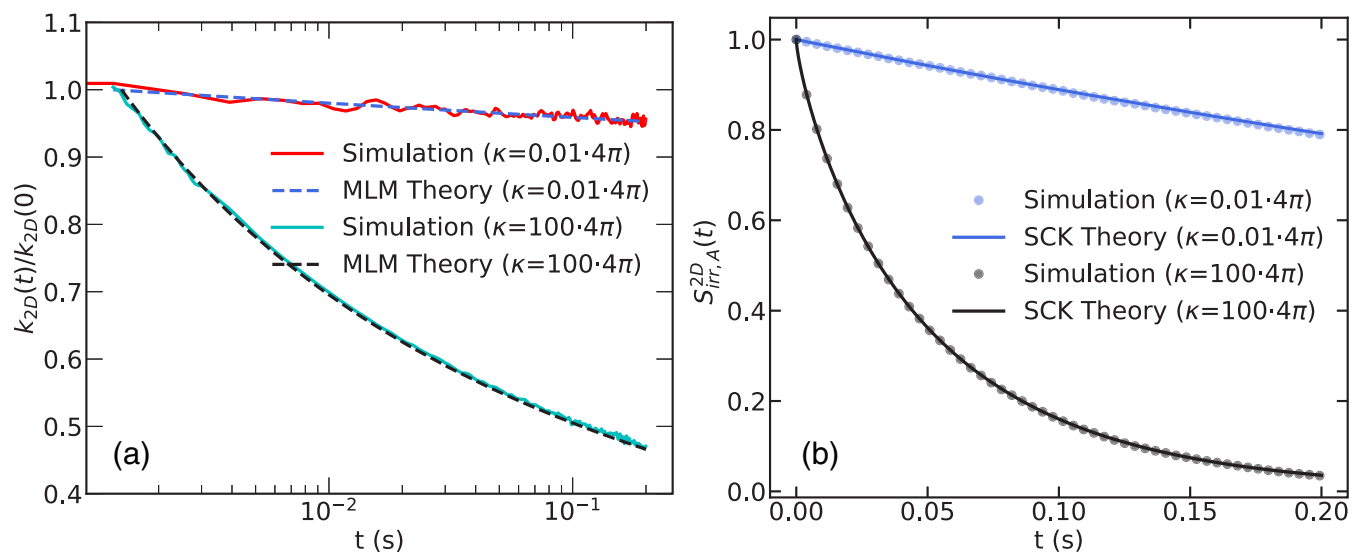

FIG. 2. Comparison of on-lattice simulations with on- and off-lattice theories for surface-surface reaction $A+B \rightarrow B$. (a) Simulated on-lattice time-dependent rate coefficients (solid lines) compared with on-lattice MLM theory in Eq. (41) (dashed lines). For better visualization of the time-dependent behavior of the two extreme cases, the simulated and theoretical lines are normalized by the initial theoretical value. (b) Simulated on-lattice survival probability of $A$ (points) compared with off-lattice SCK theory in Eq. (48) (solid lines). Activation-limited $(\kappa=0.01 \times 4 \pi)$ and diffusion-limited $(\kappa=100 \times 4 \pi)$ cases are indicated by the top and bottom lines, respectively. Simulations were performed with Spatiocyte and the following parameters: Area $=(6.5 \times 6.5) \mu \mathrm{m}^{2}, R=0.01 \mu \mathrm{m}, l=0.01 \times 1.0209 \mu \mathrm{m}$, $D_{A}=1, D_{B}=0 \mu \mathrm{m}^{2} \mathrm{~s}^{-1}, N_{a}=N_{b}=423$, duration $=0.2 \mathrm{~s}$, logging interval $=10 t_{d}$.

We compared the rate coefficients from the simulations with the theoretical values from Eq. (37). Figure 2(a) displays good agreements for both at activation-limited $(\kappa=0.01 \times$ $4 \pi)$ and diffusion-limited $(\kappa=100 \times 4 \pi)$ regimes for $t \gg t_{d}$. Next, we compared the simulated survival probability of the same reaction with the continuum-based theory, where the values are numerically evaluated according to

$$
S_{\text {irr, } A}^{2 D}(t,[B])=\exp \left[-[B] \int_{0}^{t} k_{2 D}(\tau) d \tau\right] .
$$

As shown in Fig. 2(b), the simulated results overlap almost perfectly with the continuum-based theory, thus, confirming the accuracy of MLM.

In a previous $2 \mathrm{D}$ reaction study on uncrowded lattice [82], the diffusion-limited reaction kinetics follows $k_{2 D}(t) \sim$ $t^{a}$, where $a$ is a constant. The kinetics of $2 \mathrm{D}$ activationlimited reaction, however, is generally described by the rate constant of classical reaction kinetics. As demonstrated by the theoretical and simulated lattice reaction kinetics in this work, $k_{2 D}(t)$ of activation-limited reaction decays slowly [ $\kappa=$ $0.04 \pi$ in Fig. 2(a)]. Therefore, up to a certain extent, the activation-limited $k_{2 D}(t)$ can be approximated by the classical kinetics rate constant. For diffusion-limited reaction, the asymptotic logarithmic decay in $k_{2 D}(t)$ is significantly more apparent. The behavior at the intermediate time can be well approximated by $k_{2 D}(t) \sim t^{-1 / 2}$, as shown previously in Ref. [75]. In a recent work [37], the logarithmic function of the asymptotic $k_{2 D}(t)$ is further simplified to give an effective formula $k_{2 D}(t) \sim t^{c}$, where $c$ is a function of the intrinsic rate and the diffusion coefficient. The simplified form of the time-dependent rate can be relatively accurate and convenient in describing $2 \mathrm{D}$ reaction kinetics but care should be taken to ensure the assumption, the observation time regime, and the physical parameter range are satisfied. On the other hand, the lattice rate coefficient derived in this work is based on the SCK model, which is more general and valid for both activationand diffusion-limited reactions.

\section{Reversible reaction}

Accurate simulation of reversible reaction $A+B \underset{k_{d 2 D}}{\stackrel{k_{a 2 D}}{\rightleftharpoons}} C$ according to the SCK model needs to satisfy the local detailed balance. This is achieved in MLM by adopting a rate constant $k_{d 2 D}^{\prime}$ for the dissociation reaction such that the relation,

$$
\frac{k_{a 2 D}^{\prime}}{k_{d 2 D}^{\prime}}=\frac{k_{a 2 D}}{k_{d 2 D}}
$$

is satisfied.

We perform numerical simulations to confirm the ability of MLM to correctly reproduce the steady state and time-dependent behaviors in the reversible reaction. Association rates in the activation-limited $(\kappa=0.01)$ and diffusionlimited $(\kappa=100)$ cases were used in the simulation, while the dissociation rate $k_{d 2 D}$ is set to be 10 times larger than the association rate. Simulated result is compared with the MPK1 theory in Eq. (18), obtained by numerical Laplace transform. The outcome shown in Fig. 3 indicates good agreement between the simulation and theory for time scales ranging from $t_{d}$ until equilibrium.

Since reactions in MLM take place stochastically, we examine the steady-state distribution of molecule number in the reversible homodimerization reaction, $A+A \underset{k_{d 2 D}}{\stackrel{k_{a 2 D}}{\rightleftharpoons}} B$, with association and dissociation rates, $k_{a 2 D}=0.001 \mu \mathrm{m}^{2} \mathrm{~s}^{-1}$ and $k_{d 2 D}=1 \mathrm{~s}^{-1}$, respectively. The simulation schemes of homodimerization and heterodimerization reactions are identical except in their reaction probabilities, which differ by a factor of two [see Eq. (A10)]. We initialize the simulation with $169 \mathrm{~A}$ monomers in a compartment of unit volume with voxel size $l=0.01$. The steady-state distribution of the number of $B$ dimers obtained from the simulation is shown in Fig. 4. The result of the simulation is consistent with the analytical solution from the chemical master equation [Eq. (43) in Ref. [83]]. 


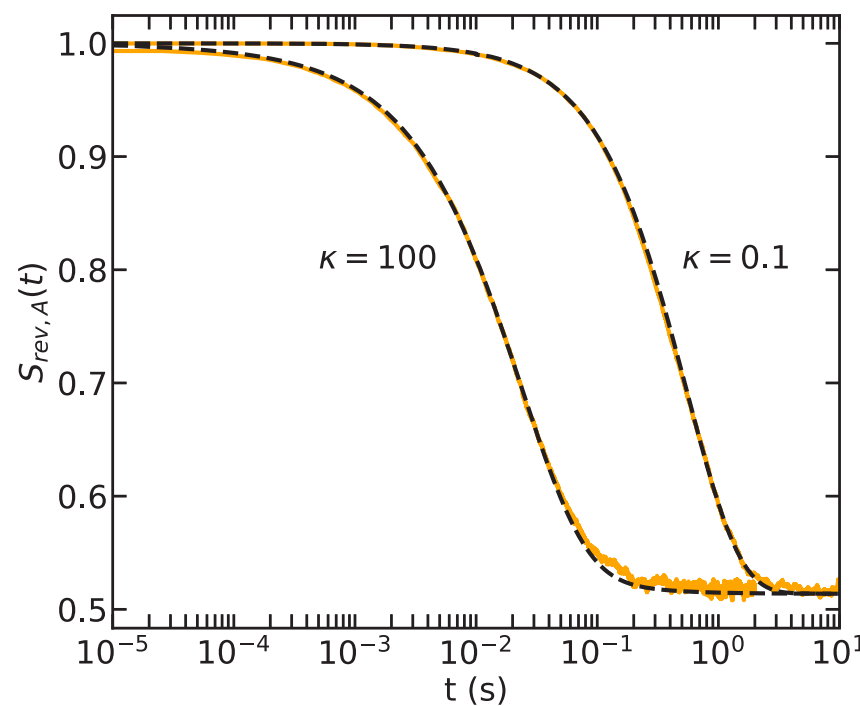

FIG. 3. Survival probability of $A$ in the surface-surface reaction $A+B \underset{k_{d 2 D}}{\stackrel{k_{a 2 D}}{\rightleftharpoons}} C$. Dashed curves are the values calculated according to the MPK1 theory given in Eq. (18); solid lines are the simulation results of Spatiocyte. Association rates in the activationlimited $(\kappa=0.1)$ and diffusion-limited $(\kappa=100)$ regimes are chosen. Simulation parameters are as follows: $k_{d 2 D}=10 k_{a 2 D}$, surface area $=(6.5 \times 6.5) \mu \mathrm{m}^{2}$ with periodic boundary, $R=0.01 \mu \mathrm{m}, l=$ $0.01 \times 1.0209 \mu \mathrm{m}, D_{A}=D_{C}=0 \mu \mathrm{m}^{2} \mathrm{~s}^{-1}, D_{B}=1 \mu \mathrm{m}^{2} \mathrm{~s}^{-1}, N_{b}=$ $20, N_{b}=401$, duration $=10 \mathrm{~s}$.

We next investigate the effects of volume exclusion on the steady-state distribution by increasing the voxel size, while retaining the number of molecules and the compartment size. With $l=0.09$, the steady-state distribution shifts to the right. This result is qualitatively consistent with the crowdinginfluenced reaction as predicted by the thermodynamic theory [84] and the master equation [83]. However, we note that the exact quantitative behavior simulated on a crowded lattice would not necessarily agree with the continuum description in all conditions because of the different degrees of molecule obstruction between on- and off-lattice representations, as demonstrated in Ref. [67]. It would require further analysis to exactly match the on-lattice crowding-influenced reaction kinetics with the continuum behavior, which is beyond the scope of the current work.

\section{Generalization of MLM theory for other lattice arrangements}

The expression of the MLM parameter derived for triangular lattice can be generalized to other lattice arrangements that adopt MLM. In general, the variable $C_{l}$ in Eq. (37) takes the form of

$$
C_{l}=4 b_{2} D \exp \left\{\left(1 / P_{a}-1\right) / b_{1}\right\} / l^{2},
$$

where $b_{1}$ and $b_{2}$ are coefficients present in the highest order term of the generating function $P\left(s_{0} \mid s_{0} ; z\right)$ :

$$
P\left(s_{0} \mid s_{0} ; z\right) \approx b_{1} \ln \left(\frac{b_{2}}{1-z}\right) .
$$

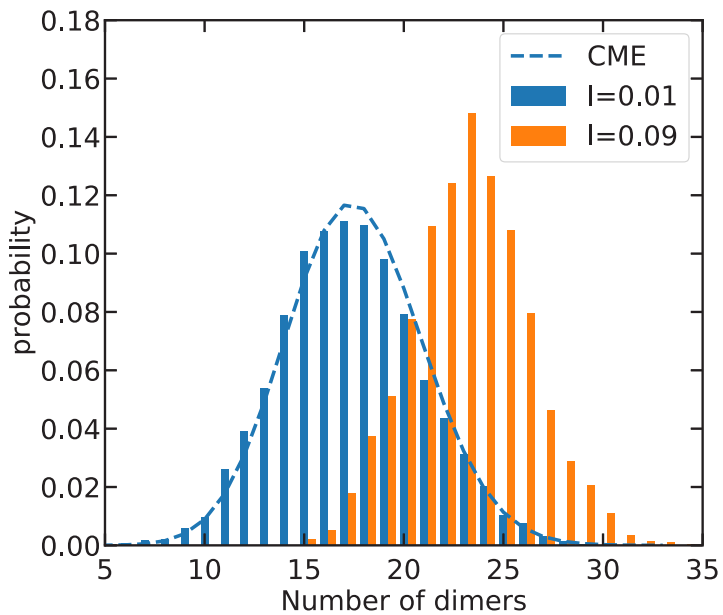

FIG. 4. Steady-state probability distribution of dimers from a reversible homodimerization reaction. The reaction is given by $A+A \underset{k_{d 2 D}}{\stackrel{k_{a 2 D}}{\rightleftharpoons}} B$, with $k_{a 2 D}=0.001 \mu \mathrm{m}^{2} \mathrm{~s}^{-1}$ and $k_{d 2 D}=1 \mathrm{~s}^{-1}$. The histogram on the left is simulated at an uncrowded condition with voxel size $l=0.01 \mu \mathrm{m}$. Dashed line is the analytical solution of the chemical master equation (CME). Histogram on the right is obtained with the same parameters except with a larger voxel size, $l=0.09 \mu \mathrm{m}$, resulting in a crowded compartment. The diffusion coefficient of $A$ is $1 \mu \mathrm{m}^{2} \mathrm{~s}^{-1}$, the length of the square compartment is $1 \mu \mathrm{m}$ and the initial number of $A$ is 169 .

On the other hand, the reaction probability has the following general form:

$$
P_{a}=\left[1+b_{1}\left(\ln \left(1 / b_{2}\right)+\frac{4 \pi D}{k_{a 2 D}}-2 \gamma\right)\right]^{-1} .
$$

The expression for the probability has the following constraint on the voxel size:

$$
l>\exp \left(\gamma-\frac{1}{2 b_{1}}+\frac{\ln b_{2}}{2}\right) R
$$

Here, as an example, we consider the square lattice, a popular lattice choice to simulate surface reactions. The coefficients for square lattice are given as $b_{1}=1 / \pi$ and $b_{2}=$ 8 [Eq. (A.187) in Ref. [65]]. The corresponding reaction probability is

$$
P_{a}=\left[1+\frac{1}{\pi}\left(\ln (1 / 8)+\frac{4 \pi D}{k_{a 2 D}}-2 \gamma\right)\right]^{-1}
$$

with the voxel size constrained by

$$
l>1.04722 R .
$$

Therefore, to recapitulate the correct continuum rate, the voxel size in square lattice has to be about 5\% larger than the molecule size. This voxel size is substantially larger than the $0.6 \%$ required by the triangular lattice. The different voxel size requirements reflect the influence of lattice arrangement on the first-passage time behavior and emphasize the importance of choosing the right MLM parameters to generate accurate reaction kinetics. 


\section{B. 1D volume-surface adsorption}

We next formulate the on-lattice 1D rate coefficient according to the SCK model and apply the rate expression to the problem of volume-surface adsorption.

In 1D lattice, the generating function for the voxel occupancy probability from origin to origin is defined as [85]

$$
P\left(s_{0} \mid s_{0} ; z\right)=\frac{1}{\sqrt{1-z^{2}}} .
$$

The corresponding first passage time distribution, obtained by the relation $F\left(s_{0} \mid s_{0} ; z\right)=1-1 / P\left(s_{0} \mid s_{0} ; z\right)$ [Eq. (I.18) in Ref. [85]), is given by

$$
F\left(s_{0} \mid s_{0} ; z\right)=1-\sqrt{1-z^{2}}
$$

Substituting Eq. (57) into Eq. (31) yields the generating function for the rebinding-time probability distribution,

$$
H\left(s_{0} \mid s_{1} ; z\right) \approx \frac{-\sqrt{2(1-z)}}{P_{a}},
$$

where we consider only the highest order term $\sqrt{1-z}$ in the limit of $z \rightarrow 1$.

The corresponding large $n$ coefficient is obtained from the generating function according to the rule given in Fig. VI.4 of Ref. [81] as

$$
H_{n}\left(s_{0} \mid s_{1}\right) \approx \frac{1}{P_{a} \sqrt{2 \pi n^{3}}} .
$$

Applying Eq. (59) to the Noyes' rate formula in Eq. (30), we obtain the asymptotic form for the $1 \mathrm{D}$ rate coefficient:

$$
k_{m}^{1 D} \approx k_{a 1 D}^{\prime} \frac{\sqrt{2}}{P_{a} \sqrt{\pi m}} .
$$

Using the definitions of the initial lattice rate constant given in Eq. (A13) and the $1 \mathrm{D}$ simulation step size $m l^{2}=2 D t$, we have the rate expression as a function of time:

$$
k_{1 D}^{\prime}(t \rightarrow \infty) \approx 2 \sqrt{\frac{D}{\pi t}} .
$$

Note that Eq. (61) shares the same time-dependent form as the continuum-based theory given in Eq. (22).

For volume-surface adsorption, the definitions for the initial adsorption rate constant in Eq. (A17) and the 3D simulation step size relation $n l^{2}=6 D t$ are used in Eq. (60). The resulting adsorption rate coefficient is given as

$$
k_{s a}^{\prime}(t \rightarrow \infty) \approx \frac{1}{2 \sqrt{2}} \sqrt{\frac{D}{\pi t}},
$$

which shares the same long-time scaling behavior with the continuum-based theory in Eq. (25) up to the same order. In contrast to the $3 \mathrm{D}$ and $2 \mathrm{D}$ cases, the long-time expression for the 1D rate coefficient does not depend on the reaction probability and the voxel size.

Since the long-time rate coefficient has the same form in both lattice and continuous spaces, we only need to match the initial lattice rate constant $k_{s a}^{\prime}$ with the adsorption rate constant $k_{s a}$ in continuum. This gives an expression for the reaction probability in terms of the adsorption rate constant, diffusion coefficient, and voxel size (derivation is shown in Appendix A 3):

$$
P_{a}=\frac{\sqrt{2} k_{s a} l}{\sqrt{3} D} .
$$

To examine the accuracy of MLM in simulating the adsorption kinetics, we performed Spatiocyte simulations using the derived expression for the reaction probability. We used a large number of cytosolic $A$ molecules in a cuboid compartment with a cross sectional area $(1 \mu \mathrm{m})^{2}$ and length $4 \mu \mathrm{m}$. An adsorbing plane is placed in the middle of the cuboid compartment, allowing adsorption from both sides of the surface. The number of adsorbed molecules at each time step is monitored.

Figure 5(a) shows the time series of $A$ on the adsorbing plane for irreversible (adsorption only) and reversible (adsorption and desorption) reactions. Simulated results agree well with the expected values according to the continuum theories for the irreversible reaction in Eq. (23) and reversible reaction in Eq. (27). The good fit can be seen at both strongly $\left(k_{s a}=500 \mu \mathrm{m} \mathrm{s}^{-1}\right)$ and weakly $\left(k_{s a}=50 \mu \mathrm{m} \mathrm{s}^{-1}\right)$ adsorbing rates. To examine the spatialtemporal concentration profile, we counted the number of cytosolic molecules near the adsorbing plane in the irreversible adsorption. The resulting concentration profile along the axis perpendicular to the adsorbing plane are shown in Fig. 5(b). The simulation results coincide very well with the curves of continuum-based theory in Eq. (26).

\section{APPLICATION OF SURFACE REACTIONS}

A cytosolic molecule can react with a membrane-bound reactant via two possible pathways: It can either perform 3D diffusion in the cytoplasm and then directly react with the membrane-bound reactant exposed to the cytosol or it can bind first to the membrane and then perform $2 \mathrm{D}$ diffusion before reacting with the reactant. Both of these pathways are often adopted simultaneously in the cell. Previous works have investigated how each pathway contributes to the overall process [86-89]. Here we apply the Spatiocyte scheme with the derived MLM expressions to simulate surface reactions comprising all dimensions. We study the contribution of each pathway to the overall reaction rate under the influence of different diffusivity and reactivity.

We consider a cuboid compartment of dimension $H \times L \times$ $L$, depicting the cytoplasmic volume. The top surface of the cuboid is reflective, whereas the bottom surface represents an absorbing lipid membrane. Each of these surfaces has the area $L \times L$. Within the system, there are two elementary species, $A$ and $B$, with radius $r=0.005 \mu \mathrm{m}$. $A_{c}$ denotes the cytosolic state of $A$ that diffuses freely in the bulk at a rate of $D_{c} . A_{c}$ can reversibly associate with the membrane to become $A_{m}$ :

$$
A_{c} \underset{k_{s d}}{\stackrel{k_{s a}}{\rightleftharpoons}} A_{m} .
$$

The ratio of the membrane association constant over the dissociation constant is the equilibrium constant, $k_{s a} / k_{s d}=$ $K_{e q}$. Upon the adsorption onto the membrane, $A_{m}$ performs 2D diffusion at a rate of $D_{m}$. On the membrane, $B$ molecules 


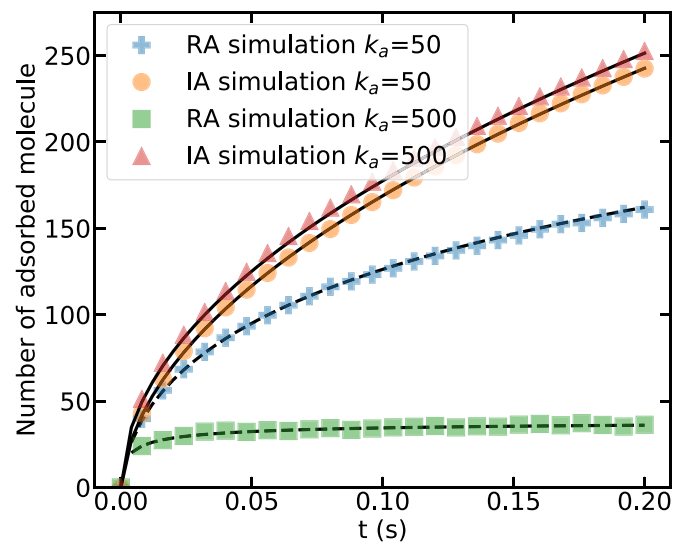

(a)

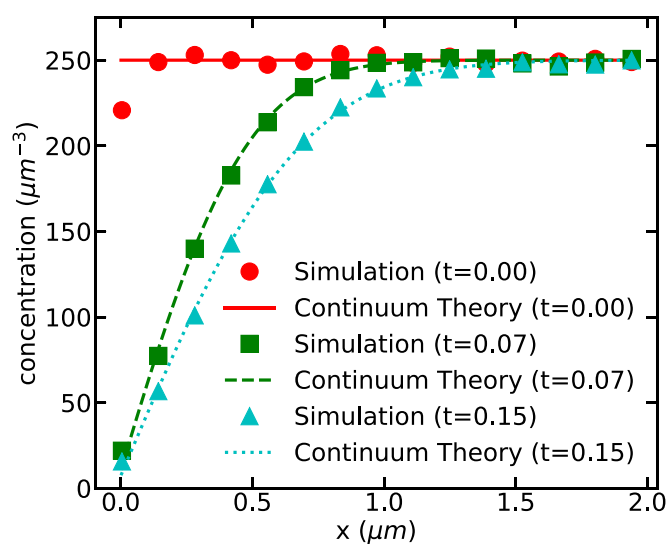

(b)

FIG. 5. (a) Time series of adsorbed molecules simulated with irreversible (IA, triangle and circle markers) and reversible (RA, plus and square markers) adsorptions. In each case, strong $\left(k_{s a}=500 \mu \mathrm{m} \mathrm{s}^{-1}\right)$ and weak $\left(k_{s a}=50 \mu \mathrm{m} \mathrm{s}^{-1}\right)$ adsorption rates were tested. In the reversible adsorption, the membrane dissociation rates are $k_{s d}=62.5$ and $6250 \mathrm{~s}^{-1}$, corresponding to the association rates $k_{s a}=50$ and $500 \mu \mathrm{m} \mathrm{s}{ }^{-1}$, respectively. Solid and dashed lines represent the continuum-based values according to the irreversible and reversible reaction formulas in Eqs. (23) and (27), respectively. (b) The concentration profile of cytosolic $A$ along the axis perpendicular to the adsorbing surface at $x=0$ for the given time points. The adsorption is irreversible with the rate $k_{s a}=50 \mu \mathrm{m} \mathrm{s}^{-1}$. Theoretical lines shown are according to the continuumbased theory in Eq. (26). Simulation parameters are as follows: $l=0.01 \mu \mathrm{m}, D_{A}=1 \mu \mathrm{m}^{2} \mathrm{~s}^{-1}$, and initial number of cytosolic molecules $N_{a}=1000$.

are initialized to be immobile and randomly distributed with concentration $[B]_{0}$.

$A$ can react with $B$ via the 3D pathway,

$$
A_{c}+B \stackrel{k_{a 3 D}}{\longrightarrow} A B
$$

or the $2 \mathrm{D}$ pathway,

$$
A_{m}+B \underset{k_{r}}{\stackrel{k_{a 2 D}}{\rightleftharpoons}} A B .
$$

$k_{a\{2 D, 3 D\}}$ denotes the intrinsic association rate constants for $2 \mathrm{D}$ and $3 \mathrm{D}$ reactions, whereas $k_{r}$ represents the dissociation rate constant.

To quantify the dominance of the $2 \mathrm{D}$ pathway, we measured the fraction of the $2 \mathrm{D}$ equilibrium rate in the total reaction rate, as in Ref. [88]:

$$
f_{2 D}=\frac{k_{\text {on } 2 D}}{k_{\text {on } 2 D}+k_{\text {on } 3 D}}=\frac{1}{1+k_{\text {on } 3 D} / k_{\text {on } 2 D}} .
$$

$k_{o n\{2 D, 3 D\}}$ represents the macroscopic effective rates for the $2 \mathrm{D}$ and $3 \mathrm{D}$ association reactions. The $k_{\text {on } 3 D} / k_{\text {on } 2 D}$ ratio is calculated using the simulated equilibrium concentrations according to the formula,

$$
\frac{k_{\text {on } 3 D}}{k_{\text {on } 2 D}}=\frac{1}{\left[A_{c}\right]_{e q}}\left(\frac{k_{r}[A B]_{e q}}{k_{a 2 D}[B]_{e q}}-\left[A_{m}\right]_{e q}\right),
$$

which is derived by solving the rate equations for Eqs. (66) and (65) at equilibrium.

We examined the dominance of the 2D pathway with changes in $D_{c} / D_{m},[B]_{0}$, and the association reaction probability, $P_{a\{2 D, 3 D\}}$ for the $2 \mathrm{D}$ and $3 \mathrm{D}$ pathways. We fixed other variables such as the sizes of the system and molecule, $k_{r}, K_{e q}$, $k_{a 2 D} / k_{r}$, and the initial concentration $\left[A_{c}\right]$. We used the typical cytosolic rate for $D_{c}\left(10 \mu \mathrm{m}^{2} \mathrm{~s}^{-1}\right)$ with $D_{c} / D_{m}$ ratio ranging from 1 to $1000 . K_{e q}=0.15 \mu \mathrm{m}$ and $k_{s d}=10 \mathrm{~s}^{-1}$ are within the biologically realistic values $[90,91]$.
From the simulation results in Fig. 6, we can observe the overall decreasing trend of $f_{2 D}$ as the ratio $D_{c} / D_{m}$ increases. The exact value of $f_{2 D}$ depends on the reaction probability and the concentration of reactant $[B]_{0}$. When the association reaction is diffusion limited $\left(P_{a 2 D}=P_{a 3 D}=1\right)$ and the reactant concentration is low $\left([B]_{0}=100 \mu \mathrm{m}^{-2}\right), f_{2 D}$ becomes more than $50 \%$ for $D_{c} / D_{m}$ between 1 and 30 . When $D_{c} / D_{m}>30$, the $3 \mathrm{D}$ pathway becomes dominant instead. At very high $[B]_{0}$ (500 $\mu \mathrm{m}^{-2}$ ), the $3 \mathrm{D}$ pathway is dominant for all ratios of

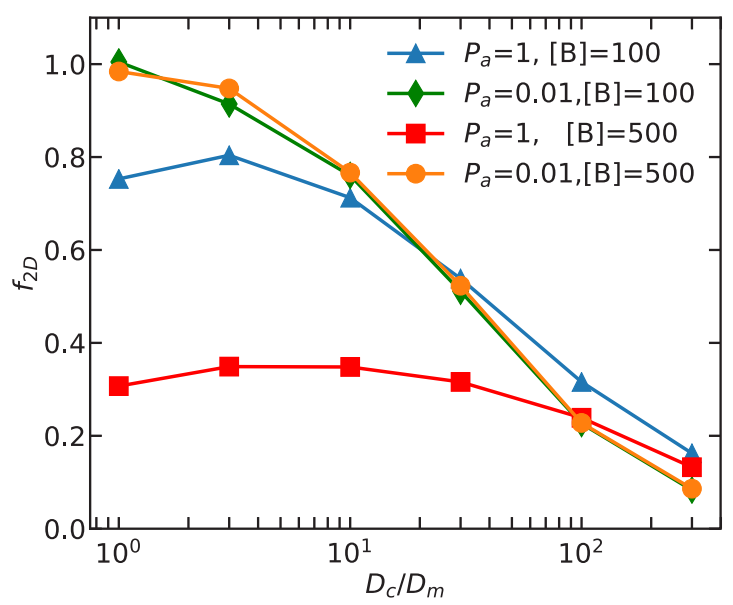

FIG. 6. Contribution of 2D reaction pathway in surface reactions. The fraction of $2 \mathrm{D}$ reaction pathway that contributes to the overall surface reaction is indicated by $f_{2 D}$ and is plotted against $D_{c} / D_{m}$. The fraction is obtained at varying reaction probabilities, $P_{a}=P_{a 2 D}=P_{a 3 D}$ and concentration of the membrane-associated reactant [B] (unit $\mu \mathrm{m}^{-2}$ ). Simulation parameters are as follows: $R=0.01 \mu \mathrm{m}, l=0.01 \times 1.0209 \mu \mathrm{m}, L=1 \mu \mathrm{m}, H=2 L, D_{c}=$ $10 \mu \mathrm{m}^{2} \mathrm{~s}^{-1},\left[A_{c}\right]=5 \mu \mathrm{M}=3000 \mu \mathrm{m}^{-3}, K_{e q}=0.15 \mu \mathrm{m}, k_{s d}=10$ $\mathrm{s}^{-1}, k_{a 2 D} / k_{r}=0.001 \mu \mathrm{m}^{2}$. 
$D_{c} / D_{m}$. When the association reaction is activation limited $\left(P_{a 2 D}=P_{a 3 D}=0.01\right), f_{2 D}$ is still larger than $50 \%$ for $D_{c} / D_{m}$ in the range $[1,30]$, and becomes less than $50 \%$ when the ratio is higher than 30 . However, unlike in the diffusion-limited case, $f_{2 D}$ in activation-limited reaction is less sensitive to the changes in $[B]_{0}$.

In typical cells, membrane-associated molecules diffuse 10-100 times slower than their cytosolic counterpart. In such a condition, our simulation results imply the following: The 2D reaction pathway will dominate the overall reaction, provided the concentration of membrane-associated reactant is low, its diffusion on the membrane is fast, and the reaction is activation limited. Conversely, the 3D reaction pathway will become dominant when the diffusion of membrane species is slow or when the membrane-associated reactant is abundant and reacts with high probability upon collision.

\section{DISCUSSION AND CONCLUSION}

MLM surface reactions have not been verified in terms of their consistency with continuum-based theory. To address this issue, we used the theoretical framework of MLM [56] to derive the correct expressions for 2D surface-surface reaction and $1 \mathrm{D}$ volume-surface adsorption on lattice. By employing the SCK model and the random walk theory, we showed that the 2D lattice reaction exhibits the same long-time behavior as the continuum-based theory. After equating the onlattice rate expression with that of the continuum theory, we obtained the formula for the reaction probability in terms of physical and lattice parameters.

Furthermore, the positively valued reaction probability imposes an additional constraint on the voxel size: It should be larger than the molecule at least by about $0.6 \%$ for the triangular lattice and by $5 \%$ for the square lattice. These constraints also meet the minimum voxel size requirement of the corresponding lattice arrangement in 3D [56]. If the voxel size is exactly the same as the molecule, the simulated timedependent reaction kinetics will deviate from the expected behavior in continuum. Such deviations should be carefully considered especially when simulating reactions containing nonlinear terms.

In $2 \mathrm{D}$ reversible reaction, we showed that correct equilibrium and time-dependent behaviors can be achieved by dissociating the substrate into an in-contact pair of product molecules, with a rate constant satisfying the local detailed balance. In 1D volume-surface adsorption, the long-time asymptotic behavior of MLM has the same form as in the continuum-based theory. The Spatiocyte scheme also generated spatiotemporal adsorption kinetics that is consistent with continuum theory when the correct expression for reaction probability was used.

Finally, we studied the contribution of a 2D reaction pathway in a surface reaction model with Spatiocyte simulations. We found that the dominant surface reaction pathway can be sensitive to the surface reactant concentration, intrinsic reaction rate, and the relative diffusivity of reactants between the bulk and the surface. For example, the 2D reaction pathway would play a significant role in regulating the overall rate for a system that has a sparse membrane-associated reactant with activation-limited rate constants.
The main advantage of MLM when modeling intracellular reaction-diffusion processes is its ability to capture the microscopic properties of molecules directly without incurring high computational cost. As an illustration, it only takes minutes for Spatiocyte to simulate thousands of molecules with a time step of $\mu$ s for a duration of seconds on a single CPU core (see performance in Ref. [56]). Spatiocyte takes physical quantities comprising molecule size, diffusion coefficient, and intrinsic reaction rate as input, and generates time-series output such as molecule copy number and trajectory.

At present, Spatiocyte supports surface reactions with various geometries at the cellular scale. It has been successfully used to capture the influence of microscopic effects on the behavior of cells at the macroscopic scale. These include the formation of a high density ring over the entire bacterium cell membrane as a result of transient membrane association and rebinding of proteins [57], the clustering of proteins on the red blood cell membrane from oxidative stress [61], and the oligomerization of receptors and its influence on ligand binding kinetics [92]. As the spatiotemporal resolution of imaging techniques continues to advance [93], timedependent reaction kinetics and molecular trajectories will become more accessible. These high resolution experimental data coupled with efficient microscopic simulation techniques such as MLM will provide a complementary way to investigate mechanisms underlying various biological reactiondiffusion processes.

The uniform voxel size adopted by MLM reduces computational complexity and consequently, contributes to its low computational cost. However, in surface systems requiring realistic simulation of distinct-sized molecules with nonspherical structures, additional considerations would be needed for MLM to be applied. One potential solution is to reduce the voxel size and let a single molecule occupy more than one voxel according to its size and shape. Alternatively, we can represent molecules with distinct shapes and sizes off-lattice and perform hybridized simulation with on-lattice molecules. The implementation and accuracy of such schemes compared to fully off-lattice methods would require further examination. Another future milestone for MLM is to establish and verify its consistency in highly crowded environments. The on-lattice rate has to be reformulated to account for the manyparticle interaction. The resulting lattice theory should then be compared and matched with the continuum-based theory.

\section{ACKNOWLEDGMENTS}

We thank K. Nishida for technical advice and support, and S. Kato for technical support and E-Cell 4 feature development. W.-X.C. acknowledges RIKEN for financially supporting his doctoral research as an International Program Associate. Part of this work was supported by JSPS KAKENHI Challenging Research (Pioneering) Grant No. 18H05371 to S.N.V.A.

W.-X.C., K.K., K.T., and S.N.V.A. designed research; W.X.C. performed research; W.-X.C., K.K., M.W., S.V.M., and S.N.V.A. analyzed data; and W.-X.C. and S.N.V.A wrote the manuscript. All authors read and commented on the manuscript. 


\section{APPENDIX A: LATTICE INITIAL RATE CONSTANT}

In this section, we provide the derivation for the initial rate constant on lattice for heterodimerization and homodimerization reactions. We describe the solutions for triangular and square lattices. Consider two reacting species $A$ and $B$, where $A$ molecules are stationary and $B$ are diffusing with relative diffusion coefficient $D$. The two species associate irreversibly to form a complex with an initial rate constant $k_{a}^{\prime}: A+B \stackrel{k_{a}^{\prime}}{\rightarrow} C$. The number of reactions that occur in the simulation time interval $t^{\prime}$ in the continuum can be approximated by the law of mass action, and is related to the lattice space by

$$
\begin{aligned}
\Delta[C]= & k_{a}^{\prime}[A][B] t^{\prime} \\
= & \text { number of reactions on lattice as a function } \\
& \quad \text { of reaction probability }
\end{aligned}
$$

where [ ] denotes the species concentration.

\section{Initial rate constant for $2 \mathrm{D}$ reaction}

On 2D lattice, the number of reactions in the interval $t^{\prime}$ according to the continuum-based framework is given as

$$
\Delta N_{C}=\frac{k_{a 2 D}^{\prime} N_{A} N_{B} t^{\prime}}{S},
$$

where $N_{i}$ is the number of molecules of species $i, \Delta N_{C}$ is the changes in molecule number $N_{c}$, and $S$ is the surface area.

The number of reactions in a step interval $t^{\prime}$ on lattice can be estimated as

$$
\Delta N_{C}=\frac{P_{a}^{\prime} N_{B} N_{A}}{N_{s v}}
$$

where $N_{s v}=S d /\left(\pi l^{2} / 4\right)$ is the number of surface voxels and $d$ is the packing density of the lattice type, and $P_{a}^{\prime}=P_{a} \alpha$ is the actual reaction probability during the encounter.

In the activation-limited scheme (see Sec. II.B. in Ref. [56] for a detailed description of the scheme), we have $t^{\prime}=t_{d}$ and $P_{a}^{\prime}=P_{a}$. Thus,

$$
\begin{aligned}
k_{a 2 D}^{\prime} & =\frac{P_{a} S}{t_{d} N_{s v}}, \\
& =\frac{\pi P_{a} D}{d} .
\end{aligned}
$$

Note that the physical unit of $k_{a 2 D}^{\prime}$ is $\left[\mathrm{L}^{2} \mathrm{~T}^{-1}\right]$, which is consistent with the continuum rate constant. For triangular lattice we have $d=\pi \sqrt{3} / 6$ and $N_{s v}=2 S /\left(\sqrt{3} l^{2}\right)$. Hence, the lattice initial rate is given as

$$
k_{a 2 D}^{\prime}=2 \sqrt{3} P_{a} D
$$

and is valid for both the activation-limited and diffusioninfluenced schemes. For square lattice, we have $d=\pi / 6$, and the initial rate is

$$
k_{a 2 D}^{\prime}=6 P_{a} D .
$$

In homodimerization reaction $A+A \stackrel{k_{a}^{\prime}}{\rightarrow} C$, the number of reactions according to continuum framework is

$$
\Delta N_{C}=\frac{k_{a 2 D}^{\prime} N_{A}\left(N_{A}-1\right) t^{\prime}}{S},
$$

whereas on lattice we have

$$
\Delta N_{C}=\frac{P_{a}^{\prime} N_{A}\left(N_{A}-1\right)}{2 N_{s v}} .
$$

From these two equations, the lattice rate constant is derived as

$$
\begin{aligned}
k_{a 2 D}^{\prime} & =\frac{P_{a} S}{2 t_{d} N_{s v}}, \\
& =\frac{\pi P_{a} D}{2 d},
\end{aligned}
$$

which differs from Eq. (A4) by a factor of 2. For the triangular lattice, the rate constant is given as

$$
k_{a 2 D}^{\prime}=\sqrt{3} P_{a} D
$$

where the relative diffusion coefficient $D$ is defined as the sum of the two diffusion coefficients $D_{A}$.

\section{Initial rate constant for $1 D$ reaction}

The number of reactions in interval $t^{\prime}$ according to the continuum framework is given as

$$
\Delta N_{C}=\frac{k_{a 1 D}^{\prime} N_{A} N_{B} t^{\prime}}{L},
$$

where $L$ denotes the length of the $1 \mathrm{D}$ system.

To be compatible with the continuum framework, we have the following assumptions in the derivation of the lattice rate constant: (i) Each voxel can accommodate more than one molecule; (ii) $A$ molecules are static whereas $B$ are mobile with a relative diffusion coefficient $D$. Then the number of reactions that takes place in a simulation step on lattice can be approximated by

$$
\Delta N_{C}=\frac{P_{a} N_{A} N_{B}}{N_{L}},
$$

where $N_{L}=L / l$ denotes the number of lattice voxels in length $L$.

Finally, the initial lattice rate constant is given by

with unit $\left[\mathrm{LT}^{-1}\right]$.

$$
\begin{aligned}
k_{a 1 D}^{\prime} & =\frac{P_{a} l}{t_{d}}, \\
& =\frac{2 D P_{a}}{l},
\end{aligned}
$$

\section{Initial rate constant for volume-surface adsorption}

Consider a cuboid compartment with an adsorbing plane placed at its center. Molecules $A$ diffuse in the bulk with a diffusion coefficient $D_{A}$. Adsorption occurs on both sides of the plane. According to the continuum theory, the number of adsorbed molecules in time step $t^{\prime}$ is approximated by

$$
\Delta N_{s}=\frac{2 k_{s a}^{\prime} N_{A} t^{\prime} S}{V},
$$

where $N_{s}$ is the number of molecules adsorbed, $N_{A}$ is the initial number of molecules in the bulk, $S$ is the area of the plane, and $V$ is the volume of the cuboid compartment.

In the case of hcp lattice, the number of adsorptions to the plane is approximated by

$$
\Delta N_{s}=P_{a} \frac{2 N_{s v}}{N_{v}} \frac{3}{12} N_{A},
$$

where $N_{s v}$ is the number of surface voxels (triangular lattice), $N_{v}=\sqrt{2} V / l^{3}$ is the number of volume voxels (hcp lattice), and $P_{a}$ is the reaction probability. Note that $3 / 12$ is the 
probability that a molecule adjacent to the plane hops onto the plane in one step and $2 N_{s} / N_{v}$ is the probability of a randomly distributed molecule $A$ is located adjacent to the plane.

By equating these two expressions and solving for $k_{s a}$, we obtain

$$
k_{s a}^{\prime}=\frac{P_{a} l}{2 \sqrt{6} t_{d}}
$$

Finally, with the diffusion time step definition $t_{d}=l^{2} / 6 D_{A}$, the initial lattice adsorption rate constant is expressed as

$$
k_{s a}^{\prime}=\frac{\sqrt{3} P_{a} D_{A}}{\sqrt{2} l},
$$

where the unit is $\left[\mathrm{LT}^{-1}\right]$.

\section{APPENDIX B: DERIVATION OF GENERATING FUNCTION}

\section{2D rebinding-time probability distribution function}

First, we express the generating function $H\left(s_{0} \mid s_{1} ; z\right)$ as given in the main text, in the following form:

$$
\begin{aligned}
H\left(s_{0} \mid s_{1} ; z\right) & =\frac{P_{a} F\left(s_{0} \mid s_{0} ; z\right)}{z+F\left(s_{0} \mid s_{0} ; z\right)\left(P_{a}-1\right)} \\
& =\frac{P_{a} F\left(s_{0} \mid s_{0} ; z\right)}{z\left[1-F\left(s_{0} \mid s_{0} ; z\right)\left(1-P_{a}\right) / z\right]} \\
& =\frac{P_{a}}{z}\left\{F\left(s_{0} \mid s_{0} ; z\right)+\frac{\left(1-P_{a}\right)}{z} F\left(s_{0} \mid s_{0} ; z\right)^{2}+\left[\frac{\left(1-P_{a}\right)}{z}\right]^{2} F\left(s_{0} \mid s_{0} ; z\right)^{3}+\ldots\right\} .
\end{aligned}
$$

Let $F\left(s_{0} \mid s_{0} ; z\right)=1-1 / P\left(s_{0} \mid s_{0} ; z\right)$ as $1-x$ and $q=1-P_{a}$, we have

$$
H\left(s_{0} \mid s_{1} ; z\right)=\frac{P_{a}}{z}\left\{1-x+\frac{q}{z}(1-x)^{2}+\left[\frac{q}{z}\right]^{2}(1-x)^{3}+\ldots\right\},
$$

in which the regular $z$ terms are neglected since $z=1$. Finally, by rearranging the generating function in terms of $x$, we obtain

$$
\begin{aligned}
H\left(s_{0} \mid s_{1} ; z\right) & =P_{a}\left\{1+q\left[1+q+q^{2}+\ldots\right]-x\left[1+2 q+3 q^{2}+\ldots\right]+x^{2} q\left[1+3 q+6 q^{2}+\ldots\right]+\ldots\right\} \\
& =P_{a}\left\{1+\frac{q}{1-q}-x \sum_{n=1}^{\infty} n q^{n-1}+x^{2} q \sum_{n=1}^{\infty} \frac{n(n+1)}{2 !} q^{n-1}+\ldots\right\} \\
& =P_{a}\left\{\frac{1}{1-q}-\frac{x}{(q-1)^{2}}-\frac{x^{2} q}{(q-1)^{3}}-\frac{x^{3} q^{2}}{(q-1)^{4}}-\ldots\right\} \\
& =P_{a}\left\{\frac{1}{1-q}-\frac{x}{(q-1)^{2}} \frac{1}{1-\frac{x q}{q-1}}\right\} \\
& =1-\frac{x}{P_{a}\left[1+\frac{x\left(1-P_{a}\right)}{P_{a}}\right]} .
\end{aligned}
$$

\section{Voxel occupancy probability on triangular lattice}

The voxel occupancy probability from origin to origin, $P_{n}\left(s_{0} \mid s_{0}\right)$ for the triangular lattice is given as [65,94]

$$
P_{n}\left(s_{0} \mid s_{0}\right)=\frac{1}{6^{n}} \sum_{j=0}^{n}\left(\begin{array}{l}
n \\
j
\end{array}\right)(-3)^{n-j} b_{j}
$$

where

$$
b_{j}=\sum_{k=0}^{j}\left(\begin{array}{l}
j \\
k
\end{array}\right)^{2}\left(\begin{array}{c}
2 k \\
k
\end{array}\right)
$$

The corresponding probability generating function is expressed as

$$
P\left(s_{0} \mid s_{0} ; z\right)=\frac{6}{\pi z \sqrt{c}} \mathbf{K}\left(k^{\prime}\right),
$$

where $c=(a+1)(b-1)$,

$$
a=\frac{3}{z}+1-\sqrt{3+\frac{6}{z}} \quad \text { and } \quad b=\frac{3}{z}+1+\sqrt{3+\frac{6}{z}}
$$


and $\mathbf{K}\left(k^{\prime}\right)$ is the complete elliptic integral of the first kind with

$$
k^{\prime}=\sqrt{\frac{2(b-a)}{c}} .
$$

Finally, the asymptotic expansion of $P\left(s_{0} \mid s_{0} ; z\right)$ in terms of the asymptotic form for $\mathbf{K}(z)$ is derived as [see Eq. (A.198) in Ref. [65]]

$$
P\left(s_{0} \mid s_{0} ; z\right) \approx \frac{\sqrt{3}}{2 \pi} \ln \left[12(1-z)^{-1}\right]\{1+O(1-z)\}
$$

[1] J. Halatek, F. Brauns, and E. Frey, Self-organization principles of intracellular pattern formation, Phil. Trans. R. Soc. B 373, 20170107 (2018).

[2] L. Wettmann and K. Kruse, The min-protein oscillations in Escherichia coli: An example of self-organized cellular protein waves, Phil. Trans. R. Soc. B 373, 20170111 (2018).

[3] Y. Zhou and J. F. Hancock, Deciphering lipid codes: K-Ras as a paradigm, Traffic 19, 157 (2017).

[4] P. N. Devreotes, S. Bhattacharya, M. Edwards, P. A. Iglesias, T. Lampert, and Y. Miao, Excitable signal transduction networks in directed cell migration, Annu. Rev. Cell Dev. Biol. 33, 103 (2017).

[5] S. Kretschmer and P. Schwille, Pattern formation on membranes and its role in bacterial cell division, Curr. Opin. Cell Biol. 38, 52 (2016).

[6] M. Pablo, S. A. Ramirez, and T. C. Elston, Particle-based simulations of polarity establishment reveal stochastic promotion of Turing pattern formation, PLoS Comput. Biol. 14, e1006016 (2018).

[7] J. Denk, S. Kretschmer, J. Halatek, C. Hartl, P. Schwille, and E. Frey, MinE conformational switching confers robustness on self-organized Min protein patterns, PNAS 115, 4553 (2018).

[8] K. Radhakrishnan, Á. Halász, M. M. McCabe, J. S. Edwards, and B. S. Wilson, Mathematical simulation of membrane protein clustering for efficient signal transduction, Ann. Biomed. Eng. 40, 2307 (2012).

[9] T. Tian, S. J. Plowman, R. G. Parton, Y. Kloog, and J. F. Hancock, Mathematical modeling of K-Ras nanocluster formation on the plasma membrane, Biophys. J. 99, 534 (2010).

[10] Y. Arai, T. Shibata, S. Matsuoka, M. J. Sato, T. Yanagida, and M. Ueda, Self-organization of the phosphatidylinositol lipids signaling system for random cell migration, PNAS 107, 12399 (2010).

[11] K. Takahashi, S. N. V. Arjunan, and M. Tomita, Space in systems biology of signaling pathways-towards intracellular molecular crowding in silico, FEBS Lett. 579, 1783 (2005).

[12] J. Berro, "Essentially, all models are wrong, but some are useful"- a cross-disciplinary agenda for building useful models in cell biology and biophysics, Biophys. Rev. 10, 1637 (2018).

[13] L. Edelstein-Keshet, Mathematical Models in Biology, Vol. 46 (Society for Industrial and Applied Mathematics, New York, 2005).

[14] J. D. Murray, Mathematical Biology (Springer-Verlag, New York, 1993).

[15] D. T. Gillespie, L. R. Petzold, and E. Seitaridou, Validity conditions for stochastic chemical kinetics in diffusion-limited systems, J. Chem. Phys. 140, 054111 (2014).
[16] S. A. Isaacson and Y. Zhang, An unstructured mesh convergent reaction-diffusion master equation for reversible reactions, J. Comput. Phys. 374, 954 (2018).

[17] S. Hellander and L. Petzold, Reaction rates for reactiondiffusion kinetics on unstructured meshes, J. Chem. Phys. 146, 064101 (2017).

[18] S. Smith and R. Grima, Spatial stochastic intracellular kinetics: A review of modelling approaches, Bull. Math. Biol., 1 (2018), 10.1007/s11538-018-0443-1.

[19] D. Fange and J. Elf, Noise-induced min phenotypes in E. coli, PLoS Comp. Biol. 2, e80 (2006).

[20] M. J. Lawson, B. Drawert, M. Khammash, L. Petzold, and T.-M. Yi, Spatial stochastic dynamics enable robust cell polarization, PLoS Comput. Biol. 9, e1003139 (2013).

[21] P. F. F. Almeida and W. L. C. Vaz, Lateral diffusion in membranes, Handbook Biol. Phys. 1, 305 (1995).

[22] E. Melo and J. Martins, Kinetics of bimolecular reactions in model bilayers and biological membranes. A critical review, Biophys. Chem. 123, 77 (2006).

[23] G. Guigas and M. Weiss, Effects of protein crowding on membrane systems, Biochim. Biophys. Acta 1858, 2441 (2016).

[24] B. C. Lagerholm and N. L. Thompson, Theory for ligand rebinding at cell membrane surfaces, Biophys. J. 74, 1215 (1998).

[25] M. Loose, E. Fischer-Friedrich, C. Herold, K. Kruse, and P. Schwille, Min protein patterns emerge from rapid rebinding and membrane interaction of MinE, Nat. Struct. Mol. Biol. 18, 577 (2011).

[26] A. Mugler, A. G. Bailey, K. Takahashi, and P. Rein ten Wolde, Membrane clustering and the role of rebinding in biochemical signaling, Biophys. J. 102, 1069 (2012).

[27] Z. Kalay, T. K. Fujiwara, and A. Kusumi, Confining domains lead to reaction bursts: Reaction kinetics in the plasma membrane, PLoS One 7, e32948 (2012).

[28] Z. Kalay, Reaction kinetics in the plasma membrane, Biotechnol. J. 7, 745 (2012).

[29] V. P. Zhdanov and F. Höök, Kinetics of enzymatic reactions in lipid membranes containing domains, Phys. Biol. 12, 026003 (2015).

[30] R. Kerketta, Á. M. Halász, M. P. Steinkamp, B. S. Wilson, and J. S. Edwards, Effect of spatial inhomogeneities on the membrane surface on receptor dimerization and signal initiation, Front. Cell Dev. Biol. 4, 81 (2016).

[31] D. V. Nicolau, J. F. Hancock, and K. Burrage, Sources of anomalous diffusion on cell membranes: A Monte Carlo study, Biophys. J. 92, 1975 (2007). 
[32] J. A. Dix and A. Verkman, Crowding effects on diffusion in solutions and cells, Annu. Rev. Biophys. 37, 247 (2008).

[33] F. Höfling and T. Franosch, Anomalous transport in the crowded world of biological cells, Rep. Prog. Phys. 76, 046602 (2013).

[34] M. J. Saxton and K. Jacobson, Single-particle tracking: Applications to membrane dynamics, Annu. Rev. Biophys. Biomol. Struct. 26, 373 (1997).

[35] K. Kaizu, W. de Ronde, J. Paijmans, K. Takahashi, F. Tostevin, and P. R. ten Wolde, The Berg-Purcell limit revisited, Biophys. J. 106, 976 (2014).

[36] A. Szabo, Theory of diffusion-influenced fluorescence quenching, J. Phys. Chem. 93, 6929 (1989).

[37] O. N. Yogurtcu and M. E. Johnson, Theory of bi-molecular association dynamics in 2D for accurate model and experimental parameterization of binding rates, J. Chem. Phys. 143, 084117 (2015).

[38] H. Berry, Monte Carlo simulations of enzyme reactions in two dimensions: Fractal kinetics and spatial segregation, Biophys. J. 83, 1891 (2002).

[39] M. J. Saxton, Modeling 2D and 3D diffusion, in Methods in Membrane Lipids (Humana Press, New York, 2007), pp. 295321.

[40] J. Schöneberg, A. Ullrich, and F. Noé, Simulation tools for particle-based reaction-diffusion dynamics in continuous space, BMC Biophys. 7, 11 (2014).

[41] F. Trovato and G. Fumagalli, Molecular simulations of cellular processes, Biophys. Rev. 9, 941 (2017).

[42] S. S. Andrews, Particle-based stochastic simulators, in Encyclopedia of Computational Neuroscience, edited by D. Jaeger and R. Jung (Springer, New York, 2018), pp. 1-5.

[43] M. Smoluchowski, Mathematical theory of the kinetics of the coagulation of colloidal solutions, Z. Phys. Chem. 92, 129 (1917).

[44] F. C. Collins and G. E. Kimball, Diffusion-controlled reaction rates, J. Colloid Sci. 4, 425 (1949).

[45] M. Doi, Stochastic theory of diffusion-controlled reaction, J. Phys. A: Math. Gen. 9, 1479 (1976).

[46] R. Erban and S. J. Chapman, Stochastic modelling of reactiondiffusion processes: Algorithms for bimolecular reactions, Phys. Biol. 6, 046001 (2009).

[47] I. C. Agbanusi and S. A. Isaacson, A comparison of bimolecular reaction models for stochastic reaction-diffusion systems, Bull. Math. Biol. 76, 922 (2013).

[48] S. S. Andrews, Accurate particle-based simulation of adsorption, desorption and partial transmission, Phys. Biol. 6, 046015 (2009).

[49] S. S. Andrews, Smoldyn: Particle-based simulation with rulebased modeling, improved molecular interaction and a library interface, Bioinformatics 33, 710 (2016).

[50] M. J. Byrne, M. N. Waxham, and Y. Kubota, Cellular dynamic simulator: An event driven molecular simulation environment for cellular physiology, Neuroinform. 8, 63 (2010).

[51] T. R. Sokolowski, J. Paijmans, L. Bossen, T. Miedema, M. Wehrens, N. B. Becker, K. Kaizu, K. Takahashi, M. Dogterom, and P. R. ten Wolde, eGFRD in all dimensions, J. Chem. Phys. 150, 054108 (2019).

[52] R. A. Kerr, T. M. Bartol, B. Kaminsky, M. Dittrich, J.-C. J. Chang, S. B. Baden, T. J. Sejnowski, and J. R. Stiles, Fast Monte Carlo simulation methods for biological reaction-diffusion sys- tems in solution and on surfaces, SIAM J. Sci. Comput. 30, 3126 (2008).

[53] J. Schöneberg and F. Noé, ReaDDy—a software for particlebased reaction-diffusion dynamics in crowded cellular environments, PLoS One 8, e74261 (2013).

[54] M. Hoffmann, C. Fröhner, and F. Noé, ReaDDy 2: Fast and flexible software framework for interacting-particle reaction dynamics, PLoS Comput. Biol. 15, e1006830 (2019).

[55] P. J. Michalski and L. M. Loew, SpringSaLaD: A spatial, particle-based biochemical simulation platform with excluded volume, Biophys. J. 110, 523 (2016).

[56] W.-X. Chew, K. Kaizu, M. Watabe, S. V. Muniandy, K. Takahashi, and S. N. V. Arjunan, Reaction-diffusion kinetics on lattice at the microscopic scale, Phys. Rev. E 98, 032418 (2018).

[57] S. N. V. Arjunan and M. Tomita, A new multicompartmental reaction-diffusion modeling method links transient membrane attachment of E. coli MinE to E-ring formation, Syst. Synth. Biol. 4, 35 (2009).

[58] S. Schnell and T. Turner, Reaction kinetics in intracellular environments with macromolecular crowding: Simulations and rate laws, Prog. Biophys. Mol. Biol. 85, 235 (2004).

[59] M. J. Saxton, A biological interpretation of transient anomalous subdiffusion. II. Reaction kinetics, Biophys. J. 94, 760 (2008).

[60] P. K. Tsourkas and S. Raychaudhuri, Monte Carlo investigation of diffusion of receptors and ligands that bind across opposing surfaces, Ann. Biomed. Eng. 39, 427 (2010).

[61] H. Shimo, S. N. V. Arjunan, H. Machiyama, T. Nishino, M. Suematsu, H. Fujita, M. Tomita, and K. Takahashi, Particle simulation of oxidation induced band 3 clustering in human erythrocytes, PLoS Comput. Biol. 11, e1004210 (2015).

[62] S. J. Lombardo and A. T. Bell, A review of theoretical models of adsorption, diffusion, desorption, and reaction of gases on metal surfaces, Surf. Sci. Rep. 13, 3 (1991).

[63] R. Imbihl and G. Ertl, Oscillatory kinetics in heterogeneous catalysis, Chem. Rev. 95, 697 (1995).

[64] J. J. Lukkien, J. P. L. Segers, P. A. J. Hilbers, R. J. Gelten, and A. P. J. Jansen, Efficient Monte Carlo methods for the simulation of catalytic surface reactions, Phys. Rev. E 58, 2598 (1998).

[65] B. Hughes, Random Walks and Random Environments: Random Walks, Vol. 1 (Clarendon, Oxford, 1995).

[66] M. Saxton, Lateral diffusion in an archipelago. The effect of mobile obstacles, Biophys. J. 52, 989 (1987).

[67] L. Meinecke and M. Eriksson, Excluded volume effects in onand off-lattice reaction-diffusion models, IET Syst. Biol. 11, 55 (2017).

[68] R. M. Noyes, A treatment of chemical kinetics with special applicability to diffusion controlled reactions, J. Chem. Phys. 22, 1349 (1954).

[69] O. G. Berg, On diffusion-controlled dissociation, Chem. Phys. 31, 47 (1978).

[70] K. R. Naqvi, K. J. Mork, and S. Waldenstrom, Diffusioncontrolled reaction kinetics. Equivalence of the particle pair approach of Noyes and the concentration gradient approach of Collins and Kimball, J. Phys. Chem. 84, 1315 (1980).

[71] M. Tachiya, Theory of diffusion-controlled reactions: Formulation of the bulk reaction rate in terms of the pair probability, Radiat. Phys. Chem. 21, 167 (1983). 
[72] A. V. Popov and N. Agmon, Exact solution for the geminate ABCD reaction, J. Chem. Phys. 117, 5770 (2002).

[73] R. H. Ritchie and A. Y. Sakakura, Asymptotic expansions of solutions of the heat conduction equation in internally bounded cylindrical geometry, J. Appl. Phys. 27, 1453 (1956).

[74] G. H. Weiss and R. J. Rubin, Random walks: Theory and selected applications, Advances in Chemical Physics, edited by I. Prigogine and S. A. Rice (John Wiley \& Sons, New York, 1982), Vol. 52, 363-505.

[75] K. R. Naqvi, J. Martins, and E. Melo, Recipes for analyzing diffusion-controlled reactions in two dimensions: Timeresolved and steady-state measurements, J. Phys. Chem. B 104, 12035 (2000).

[76] J. Sung and S. Lee, Nonequilibrium distribution function formalism for diffusion-influenced bimolecular reactions: Beyond the superposition approximation, J. Chem. Phys. 111, 796 (1999).

[77] A. V. Popov and N. Agmon, Three-dimensional simulations of reversible bimolecular reactions: The simple target problem, J. Chem. Phys. 115, 8921 (2001).

[78] J. Crank et al., The Mathematics of Diffusion (Oxford University Press, Oxford, 1979).

[79] K. Kaizu, K. Nishida, Y. Sakamoto, S. Kato, T. Niina, N. Nishida, N. Aota, M. Koizumi, and K. Takahashi, E-CELL SYSTEM version 4, GitHub Rep. (2018).

[80] M. Saxton, Anomalous diffusion due to obstacles: A Monte Carlo study, Biophys. J. 66, 394 (1994).

[81] P. Flajolet and R. Sedgewick, Analytic Combinatorics (Cambridge University Press, Cambridge, 2009).

[82] R. Grima and S. Schnell, A systematic investigation of the rate laws valid in intracellular environments, Biophys. Chem. 124, 1 (2006).

[83] C. Cianci, S. Smith, and R. Grima, Molecular finite-size effects in stochastic models of equilibrium chemical systems, J. Chem. Phys. 144, 084101 (2016).
[84] H.-X. Zhou, G. Rivas, and A. P. Minton, Macromolecular crowding and confinement: Biochemical, biophysical, and potential physiological consequences, Annu. Rev. Biophys. 37, 375 (2008).

[85] E. W. Montroll and G. H. Weiss, Random walks on lattices. II, J. Math. Phys. 6, 167 (1965).

[86] G. Adam and M. Delbrück, Reduction of dimensionality in biological diffusion processes, in Structural Chemistry and Molecular Biology, Vol. 198 (W. H. Freeman and Co., San Francisco, 1968), pp. 198-215.

[87] H. Berg and E. Purcell, Physics of chemoreception, Biophys. J. 20, 193 (1977).

[88] D. Axelrod and M. Wang, Reduction-of-dimensionality kinetics at reaction-limited cell surface receptors, Biophys. J. 66, 588 (1994).

[89] B. N. Kholodenko, J. B. Hoek, and H. V. Westerhoff, Why cytoplasmic signalling proteins should be recruited to cell membranes, Trends Cell Biol. 10, 173 (2000).

[90] R. M. Fulbright and D. Axelrod, Dynamics of nonspecific adsorption of insulin to erythrocyte membranes, J. Fluoresc. 3, 1 (1993).

[91] T. P. Tolentino, J. Wu, V. I. Zarnitsyna, Y. Fang, M. L. Dustin, and $\mathrm{C}$. Zhu, Measuring diffusion and binding kinetics by contact area FRAP, Biophys. J. 95, 920 (2008).

[92] M. Watabe, S. N. V. Arjunan, W. X. Chew, K. Kaizu, and K. Takahashi, An application of bioimage simulation: Cooperative binding measurement, arXiv:1802.10080.

[93] S. W. Hell, S. J. Sahl, M. Bates, X. Zhuang, R. Heintzmann, M. J. Booth, J. Bewersdorf, G. Shtengel, H. Hess, P. Tinnefeld, A. Honigmann, S. Jakobs, I. Testa, L. Cognet, B. Lounis, H. Ewers, S. J. Davis, C. Eggeling, D. Klenerman, K. I. Willig, G. Vicidomini, M. Castello, A. Diaspro, and T. Cordes, The 2015 super-resolution microscopy roadmap, J. Phys. D: Appl. Phys. 48, 443001 (2015).

[94] A. J. Guttmann, Lattice Green's functions in all dimensions, J. Phys. A: Math. Theor. 43, 305205 (2010). 